\title{
The XMM-BCS galaxy cluster survey
}

\section{The X-ray selected cluster catalog from the initial $6 \mathrm{deg}^{2}$}

\author{
R. Šuhada ${ }^{1,2}$, J. Song ${ }^{3}$, H. Böhringer ${ }^{1}$, J. J. Mohr ${ }^{1,2,4}$, G. Chon ${ }^{1}$, A. Finoguenov ${ }^{1,5}$, R. Fassbender ${ }^{1,5}$, S. Desai ${ }^{1,6}$, \\ R. Armstrong ${ }^{7}$, A. Zenteno ${ }^{2,4}$, W. A. Barkhouse ${ }^{8}$, E. Bertin ${ }^{9}$, E. J. Buckley-Geer ${ }^{10}$, S. M. Hansen ${ }^{11}$, F. W. High ${ }^{12}$, \\ H. Lin ${ }^{10}$, M. Mühlegger ${ }^{1}$, C. C. Ngeow ${ }^{13}$, D. Pierini ${ }^{\star}$, G. W. Pratt ${ }^{14}$, M. Verdugo ${ }^{1}$, and D. L. Tucker ${ }^{10}$ \\ ${ }^{1}$ Max-Planck-Institut für extraterrestrische Physik, Giessenbachstr. 1, 85748 Garching, Germany \\ e-mail: rsuhada@usm.lmu.de \\ 2 Department of Physics, Ludwig-Maximilians-Universität, Scheinerstr. 1, 81679 Munich, Germany \\ 3 University of Michigan, Physics Department, 450 Church Street, Ann Arbor, MI 48109-1040, USA \\ ${ }^{4}$ Excellence Cluster Universe, Boltzmannstr. 2, 85748 Garching, Germany \\ 5 University of Maryland, Baltimore County, 1000 Hilltop Circle, Baltimore, MD 21250, USA \\ ${ }^{6}$ Department of Astronomy, 1002 W. Green Street, Urbana, IL 61801, USA \\ 7 Department of Physics \& Astronomy, University of Pennsylvania, 209 South 33rd St., Philadelphia, PA 19104, USA \\ 8 Department of Physics \& Astrophysics, University of North Dakota, Grand Forks, ND 58202, USA \\ 9 Institut d'Astrophysique de Paris, UMR 7095 CNRS, Université Pierre et Marie Curie, 98 bis boulevard Arago, 75014 Paris, France \\ 10 Fermi National Accelerator Laboratory, PO Box 500, Batavia, IL 60510, USA \\ 11 University of California Observatories \& Department of Astronomy, University of California, Santa Cruz, CA 95064, USA \\ 12 University of Chicago, 5640 South Ellis Avenue, Chicago, IL 6063, USA \\ 13 Graduate Institute of Astronomy, National Central University, No. 300 Jonghda Rd, Jhongli City 32001, Taiwan \\ 14 Laboratoire AIM, IRFU/Service d'Astrophysique - CEA/DSM - CNRS - Université Paris Diderot, Bât. 709, CEA-Saclay, \\ 91191 Gif-sur-Yvette Cedex, France
}

Received 8 May 2011 / Accepted 26 October 2011

\begin{abstract}
The XMM-Newton-Blanco Cosmology Survey project (XMM-BCS) is a coordinated X-ray, optical and mid-infrared cluster survey in a field also covered by Sunyaev-Zel'dovich effect (SZE) surveys by the South Pole Telescope and the Atacama Cosmology Telescope. The aim of the project is to study the cluster population in a $14 \mathrm{deg}^{2}$ field (center: $\alpha \approx 23: 29: 18.4, \delta \approx-54: 40: 33.6$ ). The uniform multi-wavelength coverage will also allow us for the first time to comprehensively compare the selection function of the different cluster detection approaches in a single test field and perform a cross-calibration of cluster scaling relations. In this work, we present a catalog of 46 X-ray selected clusters from the initial $6 \mathrm{deg}^{2}$ survey core. We describe the XMM-BCS source detection pipeline and derive physical properties of the clusters. We provide photometric redshift estimates derived from the BCS imaging data and spectroscopic redshift measurements for a low redshift subset of the clusters. The photometric redshift estimates are found to be unbiased and in good agreement with the spectroscopic values. Our multi-wavelength approach gives us a comprehensive look at the cluster and group population up to redshifts $z \approx 1$. The median redshift of the sample is 0.47 and the median mass $M_{500} \approx 1 \times 10^{14} M_{\odot}$ $(\sim 2 \mathrm{keV})$. From the sample, we derive the cluster $\log N-\log S$ using an approximation to the survey selection function and find it in good agreement with previous studies. We compare optical mass estimates from the Southern Cosmology Survey available for part of our cluster sample with our estimates derived from the X-ray luminosity. Weak lensing masses available for a subset of the cluster sample are in agreement with our estimates. Optical masses based on cluster richness and total optical luminosity are found to be significantly higher than the X-ray values. The present results illustrate the excellent potential of medium-deep, X-ray surveys to deliver cluster samples for cosmological modelling. In combination with available multi-wavelength data in optical, near-infrared and SZE, this will allow us to probe the dependence of the selection functions on relevant cluster observables and provide thus an important input for upcoming large-area multi-wavelength cluster surveys.
\end{abstract}

Key words. large-scale structure of Universe - galaxies: clusters: general - surveys - catalogs

\section{Introduction}

The formation of the cold dark matter (CDM) dominated largescale structure of the Universe is hierarchical with smallest objects collapsing first. With passing time more and more massive structures are able to decouple from the Hubble flow and enter the non-linear regime, collapse and eventually virialize. The statistical properties of the matter density field (e.g. its power spectrum) as well as the growth of the structures are strongly

\footnotetext{
$\star$ Visiting astronomer at MPE.
}

dependent on the background cosmology and can be thus used to put constraints on cosmological models.

From this point of view, clusters occupy a very important place in the structure formation scenario, by being the most recent (i.e. redshifts $z \lesssim 2-$ coincident with the onset of the dark energy dominance) and thus also the most massive structures $\left(10^{13}-10^{15} M_{\odot}\right)$ to virialize. The cluster abundance is therefore exponentially sensitive to the growth of the large scale-structure and to the underlying cosmological parameters (Haiman et al. 2001; Majumdar \& Mohr 2003; Haiman et al. 2005). 
The key parameter in cosmological tests of this type - the total mass of clusters (identified with dark matter halos) - is itself not directly observable. Fortunately, in first approximation, clusters are virialized and their growth is gravitationally driven and therefore self-similar. This allows us to link their mass to some suitable observable quantity originating from the baryonic components of a cluster - its galaxy population and the intracluster medium (ICM). The ICM is directly observable in X-rays or through the distortion of the cosmic microwave background (CMB) imprinted by the ICM thermal electron population via inverse Compton scattering (the so-called Sunyaev-Zel'dovich effect (SZE), Sunyaev \& Zel'dovich 1972).

Since the ICM closely traces the DM potential, it offers better (i.e. lower scatter) mass-proxies than those available from optical observations of the cluster's galaxy population (e.g. Reyes et al. 2008). In X-rays, the simplest and observationally least expensive mass-proxy is the X-ray luminosity $L_{\mathrm{X}}$ (Reiprich \& Böhringer 2002; Pratt et al. 2009; Mantz et al. 2010a).

For the SZE experiments the most direct way to estimate the cluster mass is from the source signal-to-noise ratio (e.g. Williamson et al. 2011; Vanderlinde et al. 2010) and more importantly, through the integrated Compton parameter $Y_{\mathrm{SZ}}$. Numerical simulations suggest that $Y_{\mathrm{SZ}}$ is an excellent proxy of cluster mass (da Silva et al. 2004; Motl et al. 2005; Nagai 2006). First cross-comparisons with X-ray and SZE studies are generally finding good agreement between the mass estimates and no significant deviation from the self-similar predictions (Planck Collaboration 2011c,b,a; Melin et al. 2011; Andersson et al. 2011; Marrone et al. 2009; Bonamente et al. 2008).

If deeper X-ray observations are available, we can use the spectroscopic temperature $T_{\mathrm{X}}$, gas mass $M_{\mathrm{g}}$ and their combination $Y_{\mathrm{X}}=T_{\mathrm{X}} M_{\mathrm{g}}$ (the $\mathrm{X}$-ray analogue to the $Y_{\mathrm{SZ}}$ parameter, Kravtsov et al. 2006; Vikhlinin et al. 2009; Arnaud et al. 2010) as good mass proxies. Using the $Y_{\mathrm{X}}$ parameter Vikhlinin et al. (2009) put a strong constraint on the cosmological parameters including the dark energy equation of state. From a methodological point of view, this is interesting for two reasons: 1) it shows that useful cosmological constrains can be obtained already from relatively small samples of clusters of galaxies, demonstrating the exceptional potential of this type of cosmological tests; and 2) already this modest sample is practically systematics-limited, especially due to uncertainties in the mass estimation.

There are many factors that affect the scaling relations and the intrinsic scatter of the cluster populations around these relations: the presence of cool cores (Markevitch 1998; O'Hara et al. 2006; Motl et al. 2005; Pratt et al. 2009), substructures and the cluster's dynamical state (Böhringer et al. 2010; Jeltema et al. 2008) and additional non-gravitational physics (Nagai 2006), etc. In addition, one has to account for the Malmquist and Eddington bias when determining the scaling relations from an $\mathrm{X}$-ray selected sample of clusters by proper treatment of the selection and mass functions (especially for $L_{X}$, Pacaud et al. 2007; Vikhlinin et al. 2009; Pratt et al. 2009; Mantz et al. 2010a,b). As our cluster samples cover broader redshift ranges potential deviations from the self-similar evolution of the scaling relations also become an important question.

In summary, in order to be able to well constrain cosmological models with cluster samples we need: 1) large cluster samples covering redshifts beyond unity; 2) good knowledge of the cluster selection function's dependence on relevant observables and the distributions of these observables in the cluster population; 3) a reliable, low scatter mass-proxy with a known evolution in the redshift range of interest.
Surveying for clusters in SZE has a large potential with regards to all three requirements, having an almost redshift independent selection very close to a selection function with a fixed mass limit at all redshifts and a robust mass-proxy in the $Y_{\text {SZ }}$ parameter. Two ground-based large-area cluster surveys are currently underway: one by the South Pole Telescope (SPT) and one by the Atacama Cosmology Telescope (ACT). Both have already provided their first SZE-selected cluster samples (Williamson et al. 2011; Vanderlinde et al. 2010; Marriage et al. 2011; Staniszewski et al. 2009) as well as observations of already known clusters (Plagge et al. 2010; Hincks et al. 2010). Also the Planck space mission has delivered its first cluster catalog (Planck Collaboration 2011a).

While the SZE surveying approach is a very interesting new channel to perform cluster cosmology, there is still much work to be done at these early stages to understand the systematics like the influence of radio/sub-mm sources and primary CMB fluctuations on the selection, the mass calibration and sensitivity to cluster outskirts.

A multi-wavelength follow-up program of SZE selected clusters is essential, but selection function studies require also comparison of blind surveys. To this end we are conducting the XMM-BCS cluster survey. The survey field covers a $14 \mathrm{deg}^{2}$ area in the overlap region of the SPT and ACT surveys. The field has full coverage with the $4 \mathrm{~m}$ CTIO telescope at Cerro Tololo, Chile, in the framework of the Blanco Cosmology Survey (BCS) in griz bands and Spitzer observations in the mid-infrared (midIR). With this optical to mid-IR coverage we are able to provide robust photometric redshift estimates out to redshifts $\approx 0.8$ ( $\approx 1$ once also the Spitzer data is included). The X-ray coverage consists of $X M M$-Newton observations split into two distinct parts. The $6 \mathrm{deg}^{2}$ core of the X-ray survey field was observed with 42 individual, standard pointings (with $\sim 10$ ks effective exposure time). In this work, we present an initial cluster catalog based on these observations.

After SPT commenced its operations, it was soon found that the mass threshold of contemporary SZE surveys is higher than expected. In order to offer a larger overlap between the SZE and $\mathrm{X}$-ray selected cluster samples, we carried out an extension of the X-ray survey by covering an additional $8 \mathrm{deg}^{2}$ in three largearea fields utilizing the new mosaic mode type of observations. These observations allowed us to cover a significantly larger area in a very time-efficient way. First results as well as details on the analysis of this type of XMM-Newton observations are described in Šuhada et al. (2010). We demonstrate there the feasibility of blindly detecting clusters found with current generation SZE experiments in only $\sim 3 \mathrm{ks}$ long XMM-Newton observations (including tentative spectroscopic temperature measurements) in the case of two SPT detected clusters. The final $14 \mathrm{deg}^{2} \mathrm{X}$-ray cluster catalog is expected to roughly double the number of clusters in the present sample and this sample will then be interesting also for its cosmology-constraining power.

The paper is organized as follows: in Sects. 2 and 3 we describe the analysis of the X-ray observations and cluster detection pipeline. The optical data, photometric redshift estimation and spectroscopic campaign are detailed in Sect. 4. In Sect. 5 we provide our cluster sample, the physical parameters of the detected clusters and determine the survey's preliminary statistical properties. We also cross-correlate our cluster catalog with known sources and carry out a detailed comparison with the optically selected sample of Menanteau et al. (2009, and 2010, M09 and M10 hereafter). Section 6 discusses the X-ray error budget and gives an outlook on the upcoming work in the context of the XMM-BCS survey. We give our conclusions in Sect. 5. In 


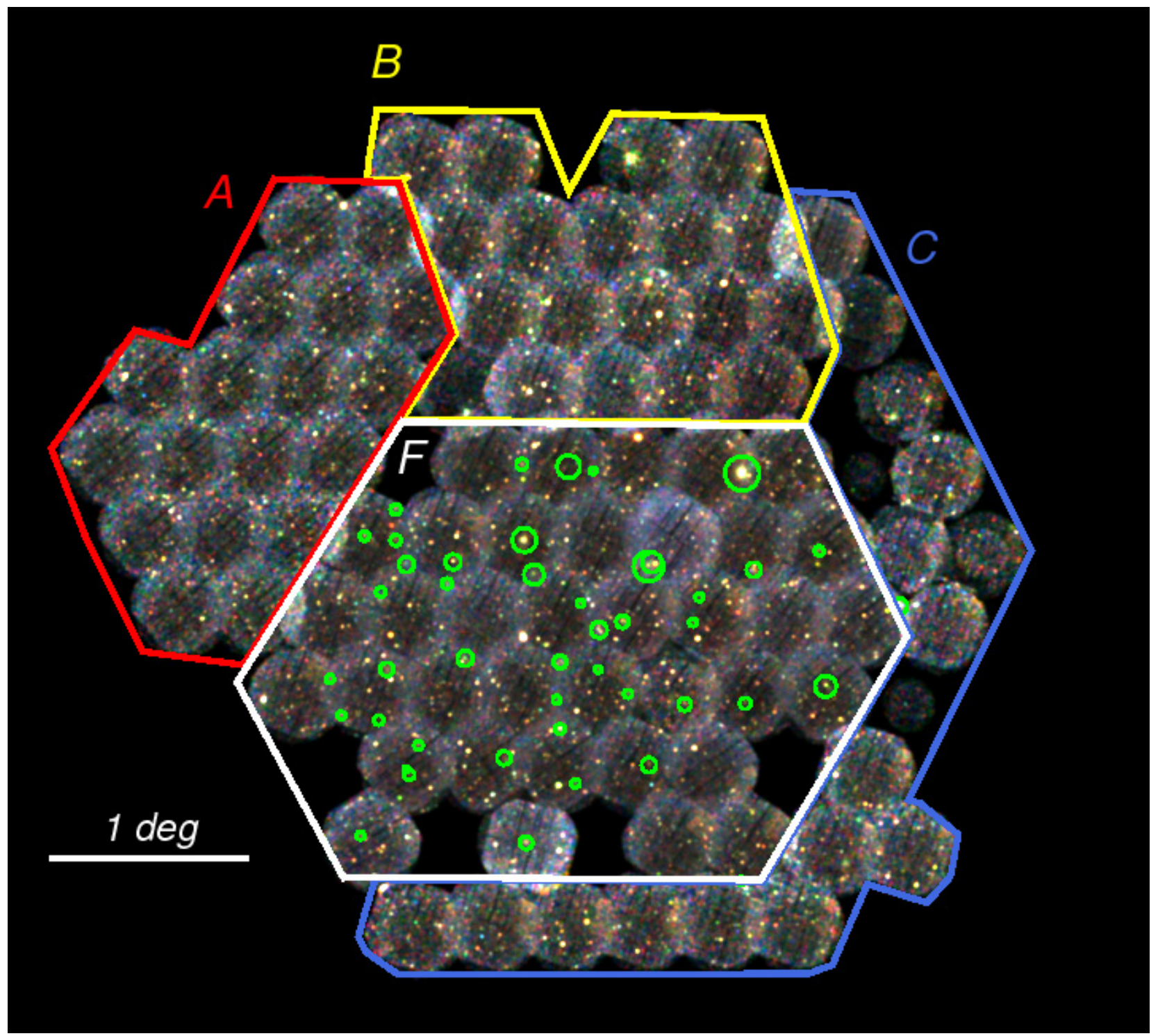

Fig. 1. Mosaic X-ray image of the $14 \mathrm{deg}^{2} X M M$-Newton sky survey. The false color image was constructed from the surface brightness in the $0.3-0.5,0.5-2.0$ and $2.0-4.5 \mathrm{keV}$ bands. The white region (F) marks the $6 \mathrm{deg}^{2}$ core of the survey presented in this work. Regions A, B and C constitute the extension of the survey by mosaic mode observations. The missing fields have significant losses due to soft proton flares. Bluer fields are affected by enhanced background. Green circles mark the positions of the present cluster sample and have a radius equal to $r_{500}$.

the appendices we provide ancillary information for the individual clusters, a preliminary comparison of our simplified sensitivity function calculations with realistic simulations and a crosscomparison with the XMM-LSS cluster survey.

We adopt a $\Lambda$ CDM cosmology with $\left(\Omega_{\Lambda}, \Omega_{\mathrm{M}}, w, H_{0}\right)=$ $\left(0.7,0.3,-1,70 \mathrm{~km} \mathrm{~s}^{-1} \mathrm{Mpc}^{-1}\right)$. Estimated physical parameters are given in apertures corresponding to overdensities by factors 200 and 500 with respect to the critical density of the Universe at the redshift of a given cluster. Throughout the article we refer to objects in our sample as "clusters" regardless of their mass. The term "group" will be used to refer to systems with masses $M_{200} \lesssim 10^{14} M_{\odot}$. We will refer to individual objects by their identification number (ID). Proper object names are listed in Table A.1.

\section{XMM-Newton data reduction}

The XMM-Newton coverage of the XMM-BCS survey core consists of 42 partially overlapping pointings with offsets of 22.8 arcmin covering a total area of about $6 \mathrm{deg}^{2}$ (see Fig. 1). The observing time was allocated in the frame of an XMM-Newton Large Program during AO6. Four additional observations were carried out in $\mathrm{AO} 7$ to replace fields with large losses due to soft-proton flaring. The observation of field F09 (Table 1) was carried out in two parts. The total observing time amounts to $\sim 580 \mathrm{ks}$, with an average total nominal time per pointing of $\sim 15 \mathrm{ks}$ (including instrument setup time and high background periods). Table 1 displays the basic information about the individual pointings. The THIN filter was used in all observations. The EPIC PN camera was operated in full frame mode.

The full XMM-BCS X-ray field is displayed in Fig. 1. The core region presented in this work is inside the white boundaries (region F). Regions A, B and C mark the three mosaic extensions of the survey. The five missing fields in region $\mathrm{F}$ have been completely lost due to flaring (F03, F05, F42) or had large time losses due to flaring and have a very high residual quiescent soft proton contamination (F07 and F13). The point source subtracted 
Table 1. The individual XMM-Newton pointings.

\begin{tabular}{|c|c|c|c|c|c|c|}
\hline \multicolumn{2}{|c|}{ Field ID } & \multirow[t]{2}{*}{ RA (J2000) } & \multirow[t]{2}{*}{ Dec (J2000) } & \multicolumn{3}{|c|}{ Exposure times $(\mathrm{ks})$} \\
\hline OBSID & Internal & & & PN & MOS 1 & MOS2 \\
\hline 0505380101 & F01 & $23: 21: 38.4$ & $-56: 07: 34.4$ & \multicolumn{3}{|c|}{ observation lost due to flaring } \\
\hline 0554561001 & F01b & $23: 22: 00.1$ & $-56: 09: 03.3$ & 7.8 & 10.4 & 10.4 \\
\hline 0505380201 & F02 & $23: 24: 23.5$ & $-56: 07: 13.2$ & \multicolumn{3}{|c|}{ observation lost due to flaring } \\
\hline 0554560201 & F02b & $23: 24: 43.8$ & $-56: 09: 03.0$ & 10.2 & 13.2 & 13.2 \\
\hline 0505380301 & F03 & $23: 27: 07.0$ & $-56: 07: 16.3$ & \multicolumn{3}{|c|}{ observation lost due to flaring } \\
\hline 0505380401 & F04 & $23: 29: 50.6$ & $-56: 07: 16.0$ & 5.2 & 6.9 & 6.9 \\
\hline 0554560901 & F04b & $23: 30: 11.7$ & $-56: 09: 01.2$ & 3.1 & 12.7 & 12.7 \\
\hline 0505380501 & F05 & $23: 32: 34.6$ & $-56: 07: 12.8$ & \multicolumn{3}{|c|}{ observation lost due to flaring } \\
\hline 0505380601 & F06 & $23: 35: 39.3$ & $-56: 08: 18.7$ & 5.6 & 10.6 & 10.6 \\
\hline 0505380701 & F07 & $23: 20: 49.3$ & $-55: 45: 35.1$ & 4.4 & 9.6 & 9.6 \\
\hline 0505380801 & F08 & $23: 23: 31.4$ & $-55: 45: 39.2$ & 9.3 & 11.0 & 9.8 \\
\hline 0505380901 & F09 & $23: 26: 12.7$ & $-55: 46: 10.2$ & 2.3 & 6.0 & 6.0 \\
\hline 0505384801 & F09b & $23: 26: 11.6$ & $-55: 46: 30.2$ & 7.3 & 9.8 & 9.8 \\
\hline 0505381001 & F10 & $23: 28: 55.3$ & $-55: 45: 39.2$ & 9.7 & 12.6 & 12.6 \\
\hline 0505381101 & F11 & $23: 31: 37.8$ & $-55: 45: 39.7$ & 7.2 & 9.7 & 9.7 \\
\hline 0505381201 & F12 & $23: 34: 19.5$ & $-55: 45: 42.6$ & 10.8 & 13.5 & 13.5 \\
\hline 0505381301 & F13 & 23:37:01.4 & $-55: 45: 39.2$ & 2.3 & 10.6 & 10.6 \\
\hline 0505381401 & F14 & $23: 19: 29.9$ & $-55: 23: 01.1$ & 10.8 & 13.9 & 13.9 \\
\hline 0505381501 & F15 & $23: 22: 09.7$ & $-55: 23: 23.1$ & 7.4 & 9.9 & 9.9 \\
\hline 0505381601 & F16 & $23: 24: 50.3$ & $-55: 23: 26.3$ & 3.2 & 11.7 & 11.7 \\
\hline 0505381701 & F17 & $23: 27: 29.7$ & $-55: 23: 45.9$ & 7.3 & 10.0 & 10.0 \\
\hline 0505381801 & F18 & $23: 30: 10.5$ & $-55: 23: 41.1$ & 11.3 & 15.2 & 15.2 \\
\hline 0505381901 & F19 & $23: 32: 51.0$ & $-55: 23: 38.5$ & 7.4 & 8.9 & 8.9 \\
\hline 0505382001 & F20 & $23: 35: 31.3$ & $-55: 23: 44.6$ & 10.5 & 13.9 & 13.9 \\
\hline 0505382101 & F21 & $23: 38: 12.0$ & $-55: 23: 43.7$ & 4.8 & 8.2 & 8.2 \\
\hline 0505382201 & F22 & $23: 18: 20.7$ & $-55: 00: 13.1$ & 11.8 & 14.3 & 14.3 \\
\hline 0505382301 & F23 & $23: 20: 58.9$ & $-55: 00: 36.3$ & 7.3 & 10.0 & 10.0 \\
\hline 0505382401 & F24 & $23: 23: 37.8$ & $-55: 00: 35.5$ & 7.5 & 10.0 & 10.0 \\
\hline 0505382501 & F25 & $23: 26: 16.6$ & $-55: 00: 42.1$ & 15.2 & 20.6 & 20.6 \\
\hline 0505382601 & F26 & $23: 28: 55.2$ & $-55: 00: 49.1$ & 9.4 & 12.1 & 12.1 \\
\hline 0505382701 & F27 & $23: 31: 34.3$ & $-55: 00: 51.0$ & 6.1 & 11.9 & 11.9 \\
\hline 0505382801 & F28 & $23: 34: 12.9$ & $-55: 00: 55.7$ & 7.1 & 9.8 & 9.8 \\
\hline 0505382901 & F29 & $23: 36: 51.9$ & $-55: 00: 54.2$ & 7.3 & 9.9 & 9.9 \\
\hline 0505383001 & F30 & $23: 19: 41.6$ & $-54: 37: 27.7$ & 12.7 & 16.4 & 16.4 \\
\hline 0505383101 & F31 & $23: 22: 18.6$ & $-54: 37: 53.3$ & 7.4 & 10.0 & 10.0 \\
\hline 0505383201 & F32 & $23: 24: 56.1$ & $-54: 37: 52.3$ & 11.6 & 13.6 & 13.6 \\
\hline 0505383301 & F33 & $23: 27: 32.7$ & $-54: 38: 04.7$ & 13.0 & 15.9 & 15.9 \\
\hline 0505383401 & F34 & $23: 30: 10.6$ & $-54: 38: 00.9$ & 9.1 & 11.9 & 11.9 \\
\hline 0505383501 & F35 & $23: 32: 29.0$ & $-54: 36: 00.3$ & \multicolumn{3}{|c|}{ observation lost due to flaring } \\
\hline 0554560601 & $\mathrm{~F} 35 \mathrm{~b}$ & $23: 32: 47.7$ & $-54: 38: 05.8$ & 7.7 & 11.4 & 11.1 \\
\hline 0505383601 & F36 & $23: 35: 25.6$ & $-54: 37: 57.3$ & 8.6 & 11.8 & 11.8 \\
\hline 0505383701 & F37 & $23: 21: 08.8$ & $-54: 15: 02.4$ & 7.5 & 9.9 & 9.9 \\
\hline 0505383801 & F38 & $23: 23: 44.6$ & $-54: 15: 01.5$ & 8.7 & 11.5 & 11.5 \\
\hline 0505384901 & F39 & $23: 25: 58.1$ & $-54: 14: 20.2$ & 5.5 & 6.8 & 6.8 \\
\hline 0505384001 & F40 & $23: 28: 56.6$ & $-54: 15: 15.2$ & 9.4 & 12.2 & 12.2 \\
\hline 0505384101 & F41 & $23: 31: 32.4$ & $-54: 15: 13.7$ & 9.9 & 12.5 & 12.5 \\
\hline 0505384201 & F42 & $23: 33: 49.9$ & $-54: 13: 13.3$ & \multicolumn{3}{|c|}{ observation lost due to flaring } \\
\hline
\end{tabular}

Notes. Quoted exposures are effective exposures with high background periods filtered out.

signal-to-noise map of the core region is displayed in Fig. 2 (see e.g. Finoguenov et al. 2009; Bielby et al. 2010).

The EPIC data was processed with the XMM-Newton Standard Analysis System (SAS) version 7.1.0 ${ }^{1}$. We reduced and calibrated the raw observational data files with the SAS tasks epchain for the EPIC PN detector and emchain for both MOS detectors. Events in bad pixels, bad columns and close to the chip gaps are excluded from further analysis.

The event lists were screened for high background periods caused by soft proton flares with a two-step sigma clipping algorithm (Pratt \& Arnaud 2003). We reject time intervals with background count rates above the $3 \sigma$ limit from the mean level in the $12-14 \mathrm{keV}$ band for PN and 10-12 keV band for MOS1 and MOS2. The mean background count rate is determined by fitting a Gaussian model to the distribution of counts in the light curve binned in $100 \mathrm{~s}$ intervals. After this first cleaning step, we apply the same $3 \sigma$ clipping procedure in the $0.3-10 \mathrm{keV}$ band on $10 \mathrm{~s}$ binned light curves to conservatively remove time intervals affected by low energy flares. An example of a two-step cleaned light curve is displayed in Fig. 3.

\footnotetext{
1 We provide a test using the current SAS 11.0.0 in Sect. 6.1.
} 



Fig. 2. The 0.5-2 keV band signal-to-noise ratio map of the XMM-BCS core region (region F in Fig. 1) smoothed with a Gaussian of 32" width. Circles indicate the $r_{500}$ radii of the detected clusters and are labeled with the cluster ID number in the catalog. Point sources have been subtracted using the method of Finoguenov et al. (2009).

Time lost due to flaring in our observations amounts typically to $\sim 20 \%$ of the full effective observing time. Six observations of the initial fields from AO6 were too heavily affected by the flaring even after the two step cleaning. Three of these fields have been replaced by observations in $\mathrm{AO}$ (F01b, F02b, F35b); the partially lost field F04 was reobserved as well.

Detection and analysis of faint diffuse sources like clusters of galaxies in shallow surveys can be additionally affected by low energy soft protons with a roughly constant flux. This so-called quiescent soft proton background can not be detected based on light curve screening due to its small temporal variations, especially not in observations with a short duration. In order to characterize possible contamination from this part of the non-X-ray background, we applied the diagnostics developed by De Luca \& Molendi (2004), based on flux ratios inside and outside the field of view of each detector. The vast majority of fields is not contaminated by the quiescent soft proton background at all in any of the detectors. Four fields (F04, F06, F16, F25) have a slight $^{2}$ contamination with negligible effect on data analysis and

\footnotetext{
${ }^{2}$ Slight contamination means $<15 \%$ increase in the background with respect to normal levels (for details see De Luca \& Molendi 2004).
}

derived results. Fields F07, F13 have significant time losses due to flaring periods (particularly in PN) and in addition are now found to have strong residual quiescent contamination $(>30 \%)$. There is no cluster in the present sample found in these fields. The PN camera in field F32 is also significantly affected ( $39 \%$ background enhancement). We identified two clusters (ID 476 and 139) in this observation. Since the results from the PN and MOS cameras for these sources are in agreement within the error bars we conclude that our background model was able to account for the enhancement. For more details on these sources see Sect. A.2.

The double component background model (see Sect. 3.1) used for the source detection and characterization can in principle account to first order for such an enhanced background by increasing the normalization of the background model. The vignetting function of such particle background has a different shape than the vignetting of the X-ray photons, but it is known only tentatively. We expect the errors from such first order approximation to be small compared to other sources of

This enhancement is modeled in first approximation by our composite background model (see text further and Sect. 3.1). 

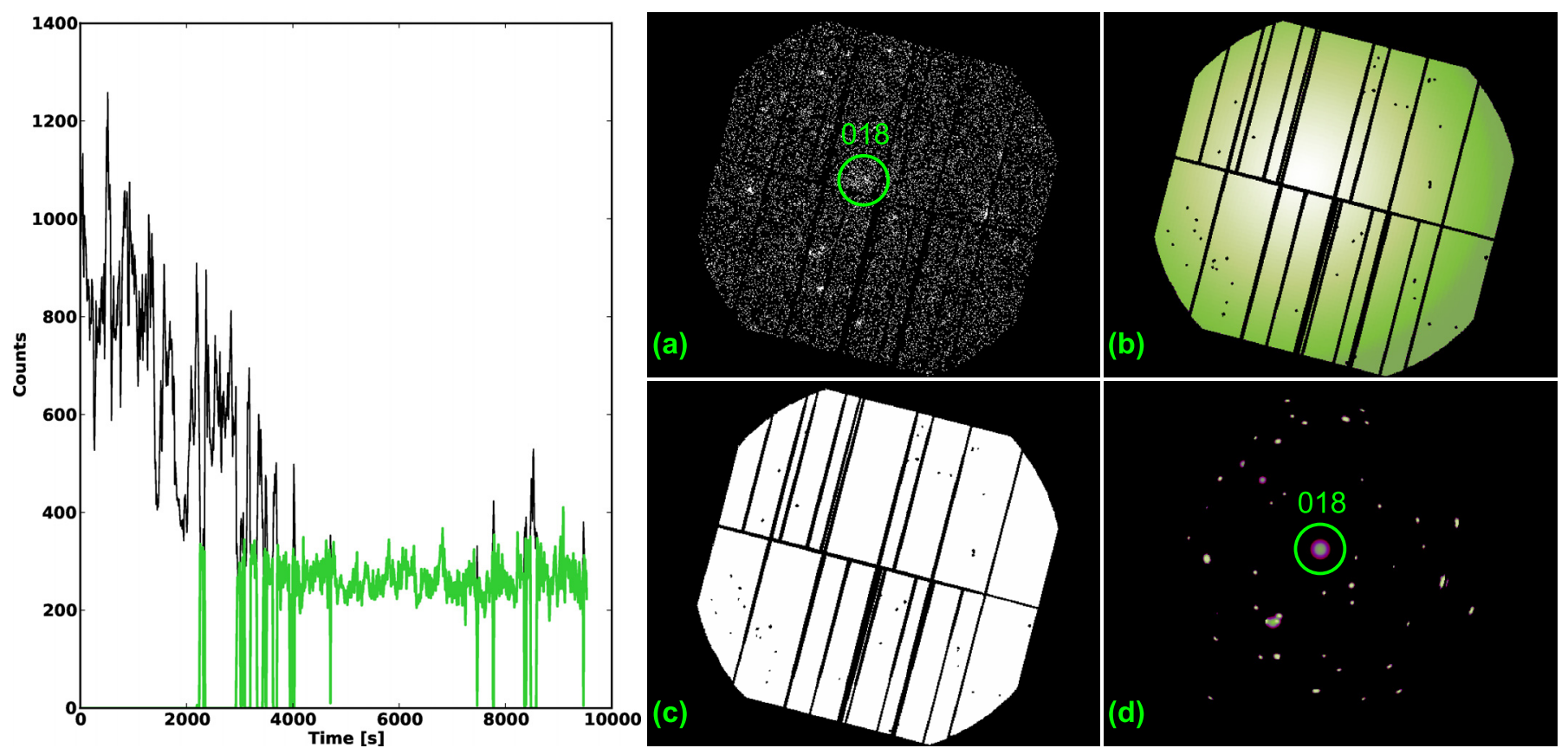

Fig. 3. Left: the black line shows the 10 second-binned PN light curve in the $0.3-10 \mathrm{keV}$ band for the field F04. The beginning of the observation was affected by flaring. The green curve shows the light curve after the two-step cleaning (see Sect. 2), which safely removed all contaminated time intervals. Right: examples of the detection pipeline products for field F04 in the $0.5-2 \mathrm{keV}$ band of the PN detector: a) counts image, b) double-component background model, c) binary detection mask, d) reconstruction of all the detected sources. The green circle ( 2 arcmin radius) marks the cluster ID 018.

uncertainty (including the shot noise). We thus decide to include into our analysis also fields with a strong residual quiescent contamination, but parameters derived for sources in these fields should be handled with caution.

We treat out-of-time-events (OOTE) for the PN detector in a standard way. For each observation, we generate an OOTE eventlist with the epchain and remove time periods identified in the two step cleaning process of the main PN eventlist. Whenever an image is extracted from the PN eventlist, we extract also an image with the same selection criteria from the OOTE eventlist, scale this image with a factor of 0.063 (full frame readout mode) and subtract it from the PN image.

\section{Source detection}

As the main source detection algorithm we utilize the sliding box technique and a maximum likelihood source fitting in their improved implementation in the SAS tasks eboxdetect and emldetect. A detailed description of the work flow and configuration of our detection pipeline, originally developed for the XMM-Newton Distant Cluster Project (XDCP), can be found in Fassbender (2008) and Fassbender et al. (2011); here we only summarize the main steps.

Source detection is carried out in three different schemes:

(i) the standard three band scheme: provides continuous, nonoverlapping coverage in three energy bands: $0.3-0.5 \mathrm{keV}$, $0.5-2.0 \mathrm{keV}$ and $2.0-4.5 \mathrm{keV}$;

(ii) the optimized single band scheme: covers the $0.35-2.4 \mathrm{keV}$ band and was chosen to maximize the signal-to-noise-ratio for clusters of galaxies with a large range of redshifts and temperatures (see also Scharf 2002). This bandpass is expected to maximize the signal-to-noise-ratio especially for high redshift systems $(z \gtrsim 1)$; (iii) the five band spectral matched scheme: uses five partially overlapping bands $(0.3-0.5,0.35-2.4,0.5-2.0,2.0-4.5$ and $0.5-7.5 \mathrm{keV})$. This scheme is equivalent to a single band detection in the full $0.3-7.5 \mathrm{keV}$ range, where the energy intervals in the overlaps have higher weighting. The shape of the weighting function roughly mimics the expected continuum spectrum shape of a hot cluster (Fassbender 2008). This setup was used only to confirm detections from the first two schemes and we do not use any results derived from it in the current work.

\subsection{Source list generation}

In order to obtain the raw source lists, we extract images from the cleaned eventlist for each detector and each band required in the given detection scheme (e.g. in the three band scheme three images for each detector, in total nine images per field). We run the sliding box detection algorithm (eboxdetect in the so-called local mode) on these images. The background for each potential source is estimated only locally in a detection cell of $5 \times 5$ pixels in 4 successive runs with the number of pixels per cell doubled in each iteration. Sources detected by this procedure are then excised, creating an image usable for proper background estimation.

We model the background of each detector and band individually with a double component background model. This background model is a linear combination of two templates based on vignetted and unvignetted exposure maps, taking into account the sky X-ray background (vignetted component) and the particle and instrumental background (unvignetted in the first approximation).

The final sliding box detection is then run utilizing the fitted background model instead of a locally estimated background. For all sources above the detection threshold we carry out a maximum likelihood fitting (with the emldetect task). A beta 

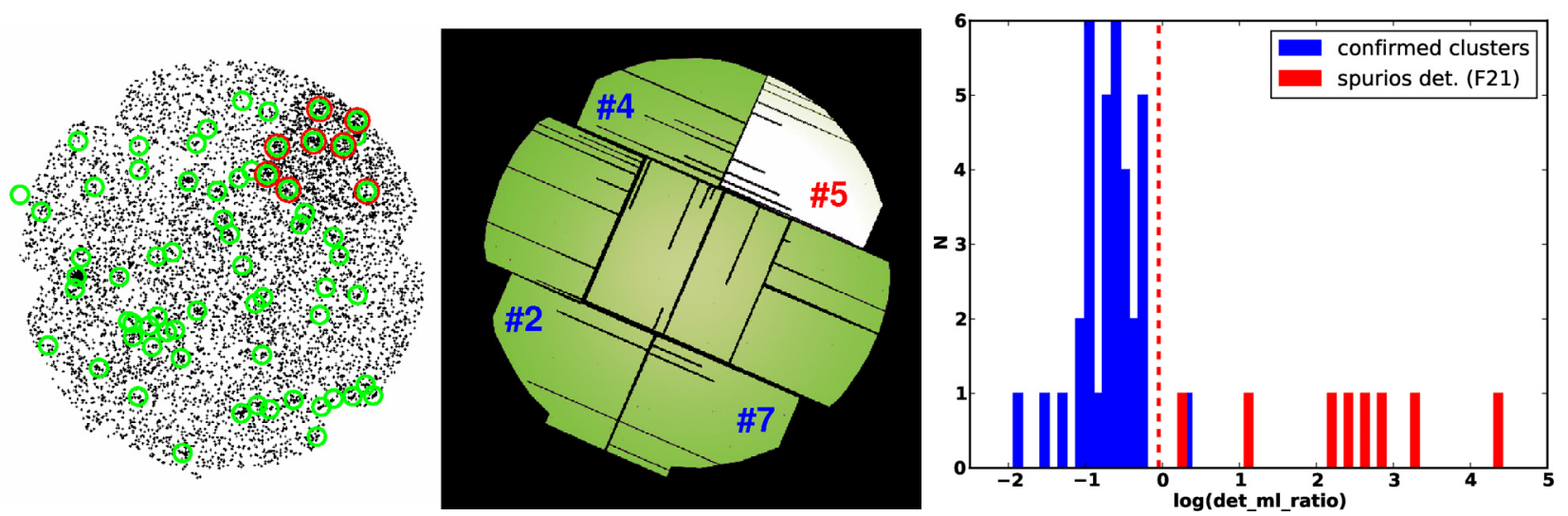

Fig. 4. Left: image of field F21 taken by the MOS2 camera in the $0.5-2.0 \mathrm{keV}$ band. The MOS2 CCD\#5 is visibly in an anomalously high ("hot") state with an enhanced background. Sources detected in this field are marked by green circles. Sources with red circles were automatically flagged as possibly spurious detections caused by the presence of the hot chip. Middle: a composite background model for the same detector and band created by fitting the double component model independently to the CCD\#5 and the rest of the chips. The three blue-marked chips are the reference chips used to identify hot chips in the observations. Right: the ratio of the total detection likelihood (log scale) from the MOS2 CCD\#5 in the $0.3-0.5$ and $0.5-2.0 \mathrm{keV}$ bands to the total detection likelihoods from all other detectors and bands (log scale). Blue bars show the confirmed clusters from our sample, the red bars the 8 flagged sources from field F21 (from the left panel). The vertical line marks where the soft band MOS2 detection constitutes $90 \%$ of the total detection likelihoods in all detectors. The flagged sources were confirmed as spurious by the optical data. A single confirmed cluster (ID 275) appears above the threshold, but is not flagged as spurious since it would have been above the detection likelihood even without the MOS2 detection (i.e. not meeting all the required criteria described in Sect. 3.1.1).

profile (Cavaliere \& Fusco-Femiano 1976) with a fixed beta value of $\beta=2 / 3$ convolved with the two dimensional pointspread function (PSF) is fitted to each source. The fit is carried out for all three detectors and all the bands in the given detection scheme simultaneously. The free parameters of the fit are the source position, normalization of the model (for each detector and band) and the core radius, $\theta_{\mathrm{c}}$, characterizing the source extent. If the extent of the source is not statistically significant, the source is refitted as a point source with extent fixed to zero.

The detection likelihood of a source is given by the det_ml parameter in the eboxdetect and emldetect tasks, defined as det_ml $=-\ln P_{\text {rand }}$, where $P_{\text {rand }}$ is the probability of observed counts arising from pure random Poissonian fluctuations. In each step of the detection process, the minimum detection likelihood is set to 6 , roughly equivalent to a $\gtrsim 3 \sigma$ detection in terms of signal-to-noise ratio.

The extent likelihood ext_ml, defined analogously to characterize the probability of the source being extended, is required to be $\geq 3$ in the three-band scheme and $\geq 5$ in the single band scheme (corresponding approximately to minimum extent significances of $\sim 2 \sigma$ and $\sim 3 \sigma$, respectively).

For a more detailed discussion and justification of the chosen detection schemes and thresholds we refer to Fassbender (2008), who also demonstrates the performance of the described source detection methods on over 450 archival XMM-Newton observations in the framework of the XDCP project. A description of the used SAS tasks can be found in the SAS 7.1.0 reference manual ${ }^{3}$.

In the current work, we aim for the best possible survey completeness including the high redshift end of the cluster distribution and reliable source classification especially close to the detection thresholds. This is also helped by combining different detection schemes and setting relatively low extent thresholds. The increasing source contamination close to the detection threshold is treated with careful screening using the optical data and ancillary X-ray information (e.g. quality flags described in Appendix A).

\footnotetext{
${ }^{3} \mathrm{xmm}$. esac.esa.int/sas/7.1.0/
}

The detected sources create a raw master list of extended source candidates. Each of these candidates is then screened visually with optical imaging data (4 band BCS imaging) and accepted to the final cluster catalog only if a significant overdensity of galaxies in the photometric redshift space is found (Sect. 4). The available Spitzer imaging for the whole field will be used in the future to confirm $z>1$ systems, where the depth of the BCS imaging is not sufficient anymore.

The purely X-ray based selection function will be developed in subsequent work based on simulations, where completeness and contamination of different detection schemes will be studied. Guided by extensive simulations of X-ray observations (Mühlegger 2010), we will get a high precision description of the survey selection function. A statistically well defined cluster sample will be drawn from the current catalog (plus its $8 \mathrm{deg}^{2}$ extension) and used to study the evolution of the cluster X-ray luminosity function and perform cosmological tests.

\subsubsection{Treatment of MOS CCDs in anomalous state}

A special note is required concerning the anomalous states of CCD\#4 of the MOS1 and CCD\#5 of the MOS2 detectors and their effect on extended source detections. Half of our fields have the MOS2 CCD\#5 in the anomalous state and $\sim 20 \%$ have an anomalous MOS1 CCD\#4 (some observations are affected by both). These anomalous ("hot") states are characterized by high overall background count rates with atypical hardness ratios. The most affected are the soft bands (in our case $0.3-0.5 \mathrm{keV}$ and 0.5-2 keV bands, see also Kuntz \& Snowden 2008).

We check for the presence of a hot chip in an observation by comparing count rates extracted from the suspected chip and the mean count rate of three other chips in symmetrical positions around the central chip (i.e. the mean count rate of $\mathrm{CCD} \# 2$, CCD\#4, CCD\#7 of MOS2 and CCD\#3, CCD\#5, CCD\#7 of MOS1). These reference chips were selected, because they best match the area, shape and position of the affected chips (see middle panel of Fig. 4). The count rates calculated in the $0.3-2.0 \mathrm{keV}$ band from the three reference chips are then 
averaged to reduce shot noise and a chip is flagged hot, if its count rate is more than $10 \%$ higher than the mean count rate from the reference chips. This threshold is chosen to be very conservative and was found to perform excellently, since chips in anomalous states have typically count rates $50-100 \%$ higher than the reference rate. The algorithm also automatically flags observations where a bright source lies on a reference chip. In these cases we manually excise the source and repeat the calculation to obtain an unaffected count rate ratio.

The exceptionally high background of the hot chips leads to many spurious extended source detections, when left untreated (see Fig. 4). We flag sources as possibly spurious detections caused by the presence of a hot chip if at the same time: 1) they lie on a chip that was flagged hot; 2) are extended; 3) the detection likelihood from the given hot MOS detector in the soft bands (sum of the $0.3-0.5$ and $0.5-2.0 \mathrm{keV}$ bands) accounts for more than $90 \%$ of the total detection likelihood and 4) the source would be under our detection threshold without the detection on the affected chip. We still visually checked every flagged source also in the optical images and confirmed the classification of these sources as spurious.

An example of this procedure can be seen in Fig. 4. The observation of field F21 has a hot MOS2 CCD\#5, clearly visible as an enhanced background (in the raw image in the left panel and in the model background in the middle). The 8 extended sources detected on this chip were flagged as potentially spurious based on the described criteria. The detection likelihood ratio (the MOS 2 detection likelihood in the soft bands over the total detection likelihood) of these 8 sources are displayed on the left panel of Fig. 4 (red) as compared to the sample of confirmed clusters in our sample (blue).

A similar criterion can be applied in principle also to spurious point source detections. An additional improvement can be achieved by weighting the input detection likelihoods by the number of pixels in the detection aperture in order to avoid a possible bias, if a source has a low detection likelihood in one of the reference detectors only because it falls on a chip gap or is (partially) out of the field-of-view.

We also make an attempt to model the high background of the hot chips by fitting the double component model to a hot chip (in first approximation) and another double component model to the remaining chips. The two parts of the background model are then combined to create a composite background map for the full detector area (middle panel of Fig. 4). All the extended sources on hot chips flagged as spurious with the described detection likelihood test, are not detected when the composite background maps are utilized, confirming the reliability of our classification. The effect of using a composite background instead of a standard background on detections coming from the remaining, nonanomalous chips is minor, since the two background models in these areas differ typically by less than $5 \%$, and only the softest bands of each detection scheme are affected. For the source characterization in observations affected by hot chips we use exclusively composite background maps.

\subsection{Growth curve analysis}

The X-ray count rate is the most direct cluster observable. With an estimate of the energy conversion factor at hand (see also Sect. 3.3) we can calculate from it the X-ray flux $F_{X}$, which in turn can be converted to X-ray luminosity $L_{\mathrm{X}}$. The luminosity is a key parameters since it allows us to calculate other important physical parameters (particularly the mass of the system) from scaling relations.

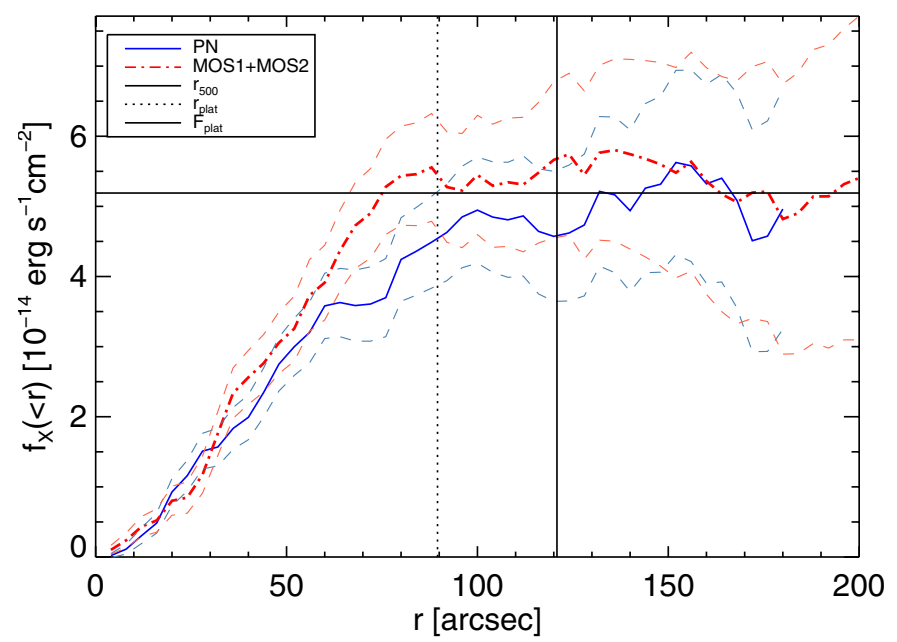

Fig. 5. Example of the growth curve analysis of source ID 018 (photo- $z=0.39$ ). The cluster's redshift and luminosity are close to the median values of the entire sample. The curves show the encircled cumulative flux as a function of radius (PN: blue curve, combined MOS: red, dot-dashed). The PN and MOS curves are in good agreement. Dashed lines mark the flux measurement error bars which include the Poisson noise and an additional 5\% systematic error from the background estimation. The estimated plateau flux is $F_{\text {plat }}=5.19 \times$ $10^{-14} \mathrm{erg} \mathrm{s}^{-1} \mathrm{~cm}^{-2}$ (horizontal line), reached at $r_{\text {plat }} \sim 90 \mathrm{arcsec}$. The vertical line signifies the estimated $r_{500}$ radius of the source, $r_{500}=$ $0.6 \mathrm{Mpc}(\sim 117 \mathrm{arcsec})$. In this case, the plateau radius is slightly smaller than $r_{500}$ and the flux and luminosity for $r_{500}$ had to be extrapolated from their plateau values. The required extrapolation is only $\sim 2 \%$ in this case. See Sect. 3.3 for details.

A typical cluster of galaxies in relatively shallow observations like ours appears as a faint diffuse source with typically $\gtrsim 100$ source photons registered (total from all three standard bands and detectors). Thus in order to get a reliable measurement of the flux and trace the emission of the cluster as far out as possible, we have to employ a robust method. In this work we utilize the growth curve method developed for the REFLEX and NORAS cluster samples derived from the ROSAT all sky survey by Böhringer et al. (2000). Here we summarize the procedure.

For each source, we extract images, exposure maps and background maps in the $0.5-2.0 \mathrm{keV}$ band, excluding all point sources detected by the pipeline. MOS 1 and MOS2 products are then directly co-added, since the difference in their response matrices is small. We run the growth curve program on the PN and co-added MOS images independently.

In this analysis we use the X-ray center coordinates obtained from the beta model fitting procedure in the source detection step. We also explored the possibility of recentering by minimizing the dipole moment of the count distribution (see e.g. Böhringer et al. 2010). This procedure usually yielded centers very close to the best-fit beta model coordinates, but for faint sources often completely diverged. The best-fit coordinates were always found to be a good description of the detected X-ray emission centroid.

Counts are extracted from the image in concentric rings starting from the center and scaled by the exposure time. In this way we obtain the total (source + background) count rate profile. The expected background count rate is estimated from the model background map and subtracted for each ring from the total count rate, obtaining the source count rate profile. The growth curve is the cumulative background subtracted source count rate profile. An example of a growth curve is displayed in Fig. 5 
(shown here in flux units using the energy conversion factor calculated as described in Sect. 3.3).

We term the radius of the full aperture inside which a stable growth curve can be obtained, the extraction radius $r_{\text {ext }}$ (typically 150-200 arcsec). It is adjusted for each source individually (increased for brightest, most extended sources or trimmed for sources close to the edge of FOV or to a partially blended systems) and includes the source itself as well as enough sky region to check the reliability of the double component background subtraction.

If the background model describes the local background accurately, the growth curve levels off to a flat plateau at the outer edge of the source. To estimate the total detected cluster emission, we first calculate the significance radius $r_{\text {sig }}$, defined as the radius outside which the source signal increases less than the $1 \sigma$ uncertainty in the count rate. The significance radius thus gives the outermost radius where the potential increase of the growth curve becomes less than $1 \sigma$ significant.

To alleviate the effect of shot noise, $r_{\text {sig }}$ is determined by smoothing the growth curve in 20 and 48 arcsec windows (5 and 12 pixels respectively). For most clusters the two estimates are in agreement. In the remaining cases, the local background usually exhibits irregular features not captured by the double component model and we select the more appropriate $r_{\text {sig }}$ and plateau after visual inspection.

In addition, a single multiplicative correction factor to the background model can be set, if the plateau exhibits a significant residual slope. This additional factor corrects the overall normalization of the double component model locally inside $r_{\text {ext }}$. The average background correction factors are $-2 \%$ (i.e. a $2 \%$ decrease compared to the default double component background) for PN and $0 \%$ for MOS (with standard deviations $7 \%$ and $8 \%$, respectively). More than $3 / 4$ of the present sample have correction factors smaller than 10\%. Reiprich (2001) and Reiprich \& Böhringer (2002) used a similar correction procedure utilizing a second order polynomial to obtain stable plateaus. In our case, a simple correction factor turned out to be sufficient and not leading to background over-fitting.

After setting the background correction, the total source count rate is estimated as the count rate of the plateau. The flat plateau of the growth curve outside $r_{\text {sig }}$ is then fitted with a line. If the slope of the line is less than $0.8 \%$ per radial bin, the plateau fit is accepted and the plateau count rate $C T R_{\text {plat }}$ is estimated as the mean of the fitted line. If the slope is still not negligible, an additional attempt is made to find a stable plateau by iteratively removing the outermost and innermost (still outside $r_{\text {sig }}$ ) bins. We note that in $\sim 80 \%$ of cases the first simple fit is fully acceptable and no further iterations are necessary. For more detailed description of the iterative process and quality flags of the plateau fit see Appendix A. The plateau radius $r_{\text {plat }}$, is defined simply as the radial distance where the growth curve first reaches $C T R_{\text {plat }}$.

We provide a performance test of our X-ray photometry method on the example of the XMM-LSS cluster catalog (Pacaud et al. 2007) in Appendix C.2. The main advantages of the growth curve method thus are: (i) excellent sensitivity allowing us to trace cluster emission to the outermost faint outskirts; (ii) it makes no assumptions about the source profile unlike methods based on beta model fitting, which is fully degenerate in the regime with $<400-500$ counts and is known not to be an appropriate description of cluster emission for irregular and cool core clusters; (iii) the method allows to check and correct the background modelling which is done for the whole field of view, by adjusting several parameters to the conditions local to each analyzed source; (iv) the PN and combined MOS growth curves are treated completely independently. Their comparison provides us with an important consistency check and allows us to treat instrument specific features in the background separately.

\subsection{Physical parameter estimation}

With a stable PN and MOS growth curve at hand we determine all the relevant physical parameters of the clusters (see Table 2) in an iterative way:

1. The physical parameters are set to their initial values $\left(r_{500}, T_{500}\right)=(0.5 \mathrm{Mpc}, 2.5 \mathrm{keV})$.

2. The physical aperture radius is converted to arcseconds using the assumed cosmological parameters. The total count rate inside this radius is estimated from the PN and MOS growth curves in the $0.5-2 \mathrm{keV}$ band.

3 . The count rates are converted to flux with an energy conversion factor (ECF) calculated assuming a MEKAL spectral model (Mewe et al. 1985; Kaastra 1992; Liedahl et al. 1995 ) with abundance set to 0.3 times solar abundance, temperature equal to the trial $T_{500}$ value and redshift set to the photo- $z$ value (or spectroscopic redshift where available).

To account for the spatial variation of the spectral response of the detectors we calculate a response matrix for each source individually in a 150 arcsec aperture centered on the source for the THIN filter. The MOS2 response matrix is used to calculate the ECF of the co-added MOS count rates.

This procedure is used to estimate the $0.5-2 \mathrm{keV}$ and bolometric fluxes for PN and MOS and the same model is also used to convert the fluxes to luminosities.

4. In some cases the estimated value of $r_{500}$ in the given iteration is larger than $r_{\text {plat }}$ (i.e. larger than the region with directly measurable emission), and therefore an extrapolation factor has to be applied to the flux and luminosity estimates. We correct for the missing flux between the plateau radius and current iteration estimate of $r_{500}$ by extrapolating the source emission assuming a beta model. The $\beta$ and $r_{\text {core }}$ parameters of the beta model are calculated using the scaling relations of Reiprich (2001; see also Reiprich \& Böhringer 2002; Finoguenov et al. 2007):

$r_{\text {core }}=0.07 \times r_{500}\left(\frac{T}{1 \mathrm{keV}}\right)^{0.63}$ and $\beta=0.4\left(\frac{T}{1 \mathrm{keV}}\right)^{1 / 3}$

The good sensitivity of the growth curve method allows us to trace cluster emission out to large radii, therefore the required extrapolation is typically minor. The mean correction is $\sim 5 \% / \sim 6 \%$ for PN/MOS ( $\sim 6 \%$ at maximum).

In cases when $r_{500} \leq r_{\text {plat }}$ no extrapolation is needed and the flux and luminosity estimates are independent of any assumption about the spatial distribution of the source emission.

5. The source flux and luminosity are then obtained by averaging the PN and MOS fluxes (luminosities) weighted by their inverse squared errors. Sources for which the PN and MOS estimates do not agree or one of the estimates is missing (e.g. source outside of the FOV of a given detector) are flagged (Table A.1). An X-ray photometric quality flag is also assigned to each source based on the quality of the plateau fit, portion of pixels outside the detection mask, presence of anomalous features in the X-ray background and visual screening.

6. We then use this total (i.e. camera averaged) bolometric luminosity value to calculate the temperature and mass of the 


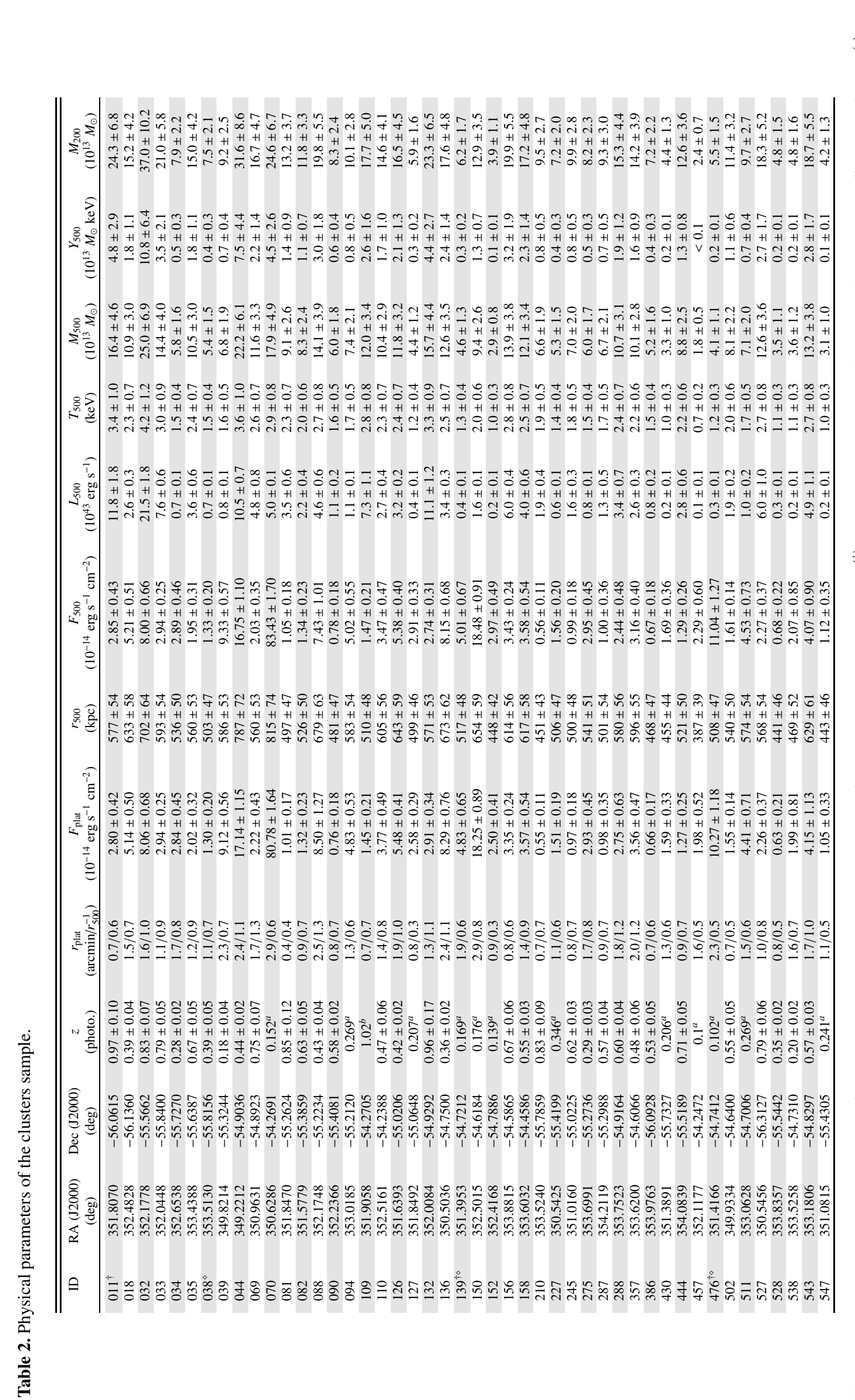

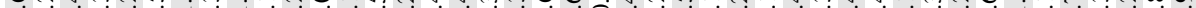
舟



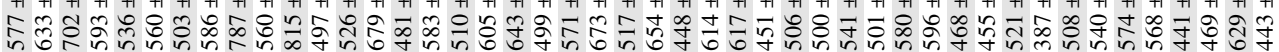

ๆำ舟年

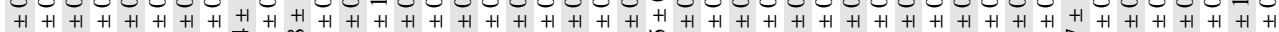

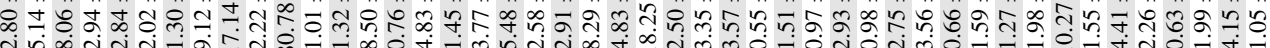

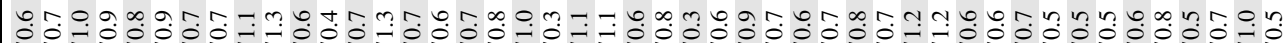



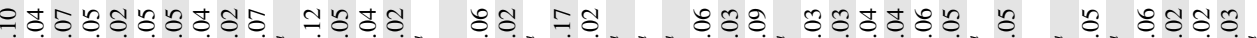

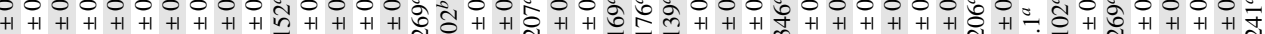

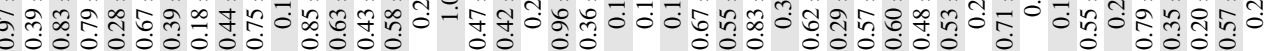

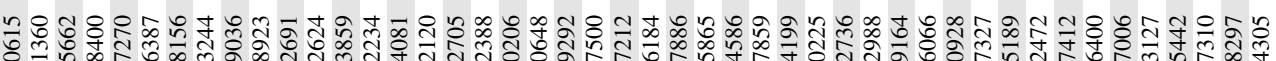

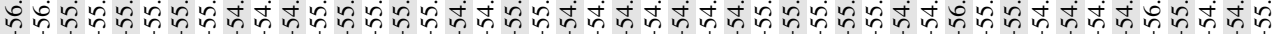


cluster for the next iteration by utilizing the $L-T$ and $L-M$ scaling relations of Pratt et al. (2009):

$M=2 \times 10^{14} M_{\odot}\left(\frac{h(z)^{-7 / 3} L}{1.38 \times 10^{44} \mathrm{erg} \mathrm{s}^{-1}}\right)^{1 / 2.08}$

$T=5 \operatorname{keV}\left(\frac{h(z)^{-1} L}{7.13 \times 10^{44} \mathrm{erg} \mathrm{s}^{-1}}\right)^{1 / 3.35}$.

These relations were obtained by the BCES orthogonal fits (Akritas \& Bershady 1996), which do not treat $T_{500}$ (or $M_{500}$ ) as the independent variable, since our independent variable is in fact $L_{X}\left(<r_{500}\right)$. At this stage it is impossible to safely detect and remove emission from possible cooling cores because of the limited resolution of XMM-Newton. Therefore we opt not to do so and use the relations that include also the core regions.

The $Y_{500}$ parameter is calculated from the Pratt et al. (2009) relation:

$Y_{\mathrm{X}}=2 \times 10^{14} M_{\odot} \operatorname{keV}\left(\frac{h(z)^{-9 / 5} L}{5.35 \times 10^{44} \mathrm{erg} \mathrm{s}^{-1}}\right)^{1 / 1.04}$.

7. The $M_{500}$ estimate is then used to obtain a new $r_{500}$ radius from $r_{500}=\sqrt[3]{3 M_{500} / 4 \pi 500 \rho_{\mathrm{C}}(z)}$, where $\rho_{\mathrm{C}}(z)$ is the critical density of the Universe at redshift $z$. This new $r_{500}$ aperture is used along the updated $T_{500}$ value to recalculate the fluxes and luminosities by repeating steps $1-7$. The process is repeated until convergence to a final solution.

The final parameters are listed in Table 2. The table also includes mass estimates in the $r_{200}$ aperture. An NFW profile (Navarro et al. 1997) was assumed in order to extrapolate the mass from the $r_{500}$ to $r_{200}$ aperture. The extrapolation factor was calculated using the approximation derived by Hu \& Kravtsov (2003), where the parameters of the NFW profile are iteratively estimated from the final (i.e. converged) $M_{500}$ mass by utilizing the Bullock et al. (2001) relation for the concentration parameter calculation.

We provide a discussion on the scaling relations utilized here and the error budget of our iterative method in Sect. 6.1.

\section{Photometric redshift estimation}

In order to measure the photometric redshifts (photo- $z s$ ) of the X-ray selected systems in our sample, we applied the redsequence redshift estimator to the Blanco Cosmology Survey (BCS) imaging data which covers two $50 \mathrm{deg}^{2}$ patches of the southern sky and includes the full area of the present XMM-Newton survey. The BCS is a 60 nights NOAO survey program carried out from 2005-2008 on the Blanco $4 \mathrm{~m}$ telescope at Cerro Tololo Interamerican Observatory. This griz survey was tuned to the required depths to follow the passively evolving cluster red sequence population to $L^{*}+1$ at $5 \sigma$ significance out to $z=1$. The data were acquired using two offset layers of imaging in $g$ and $r$ band, and three offset layers of imaging in $i$ and $z$ band.

The BCS data have been processed and calibrated using a development version of the Dark Energy Survey data management system (DESDM v4, Mohr et al. 2008). The core processing includes flat fielding, bias subtraction, illumination and fringe corrections. Astrometric refinement uses the USNO-B2 catalog and the AstrOmatic tool SCAMP (Bertin 2006). Reduced single epoch images are combined into deep, coadded images using the AstrOmatic tool SWarp together with DESDM code that homogenized the PSF to the median seeing within each tile/band combination. Model fitting photometry using bulge+disk decomposition was carried out using the extended version of SExtractor (Bertin \& Arnouts 1996) developed within DESDM to enable PSF corrected model fitting to all detected objects on an image. Photometric calibration was carried out using PSF model magnitudes calibrated using the stellar locus in the color space defined by griz $H$ where the $H$ band data from 2MASS (Skrutskie et al. 2000) provides the overall photometric scale (Armstrong et al. 2010). A similar approach has been used for the processing and calibration of all SPT cluster followup and redshift estimation using Blanco $4 \mathrm{~m}$ data, and the results have enabled detailed studies of cluster galaxy properties (Zenteno et al. 2011) as well as precise photometric redshift estimation (High et al. 2010; Williamson et al. 2011).

The full description of our photometric redshift method is provided in Song et al. (2011); here we give its brief summary. The red-sequence redshift estimator utilizes all available filters, $\left(g_{-}^{-}, r_{-}, i_{-}^{-}\right.$, and $\left.z\right)$ to search for redshift peaks in the density distribution of galaxies within a radius of $0.8 \mathrm{Mpc}$ centered on the X-ray detection. To define the red-sequence at each redshift slice, we assume a single stellar population (SSP) model by Bruzual \& Charlot (2003) with a single burst of star formation at $z_{\mathrm{f}}=3$ and passive evolution of red galaxies thereafter. The SSP models are run with six distinct metallicities in order to be able to model a tilted red sequence. The models are calibrated to reproduce the tilt of the color-magnitude relation for the Coma cluster (e.g. Bower et al. 1992).

A single stellar population (SSP) model assuming a single burst of star formation at $z_{\mathrm{f}}=3$ and passive evolution of red galaxies thereafter, by Bruzual \& Charlot (2003) is used to define the red-sequence at each redshift slice, which is calibrated to Coma cluster.

The contribution of background galaxies is estimated from a surrounding $36^{\prime} \times 36^{\prime}$ sky patch and statistically subtracted. For each X-ray cluster candidate the whole redshift range from $z=0$ to $z=1.05$ is scanned through using simultaneously two colors that bracket the $4000 \AA$ break for a given redshift. This suppresses false overdensity peaks at transitional redshifts where the $4000 \AA$ break moves between two adjacent bands (e.g. the transition between the $g$ and $r$ band around $z \approx 0.35$ ). Once a peak in redshift space is identified, we refine the redshift estimate by fitting a Gaussian function to the redshift density distribution. We then select cluster members in a stripe ( 0.05 width in color) around the estimated red-sequence. The final cluster redshift value is calculated as the inverse color error weighted mean redshift of the selected member galaxies. This assures that the reliability of the photo- $z$ values for the whole system is always better than for any individual galaxy. In a few cases two or more solutions were found by our algorithm. For these systems we visually check the obtained redshift distributions and select the more likely solution given the positions of galaxies with respect to the X-ray emission. An example of a final color- magnitude diagram is shown in Fig. 6 for cluster ID 018 (its redshift is close to the median redshift of the cluster sample).

The described photo- $z$ estimation method allows us to measure the cluster redshift with good precision up to $z \approx 0.8$ even for low richness systems. The overall uncertainty of the photo- $z s$ is on the $\sim 10 \%$ level. While care was taken to obtain reliable results also for $z \gtrsim 0.8$ systems (see Fig. 7 for two examples), here the already obtained Spitzer mid-infrared observations will 

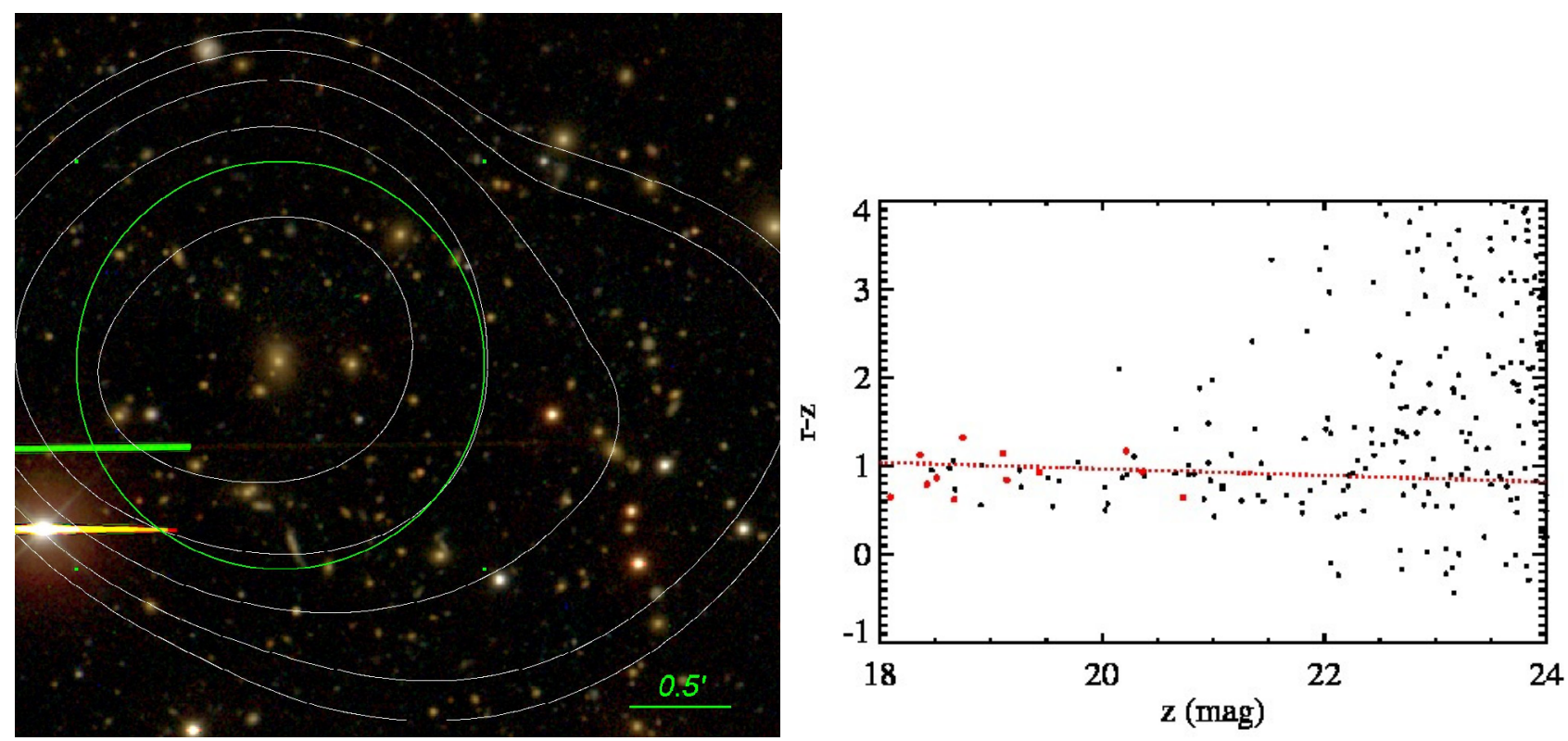

Fig. 6. Left: pseudo-color image of source ID 018 from the Blanco Cosmology Survey in the gri bands. X-ray contours are overlaid in white. The green circle shows the estimated $0.5 \times r_{500}$. Right: color-magnitude diagram $(r-z$ vs. $z)$ for the cluster ID 018 . The red points show the member galaxies used for the photo- $z$ estimate and the red dotted line indicates the single stellar population model at cluster redshift $z=0.39$ (see text for details).
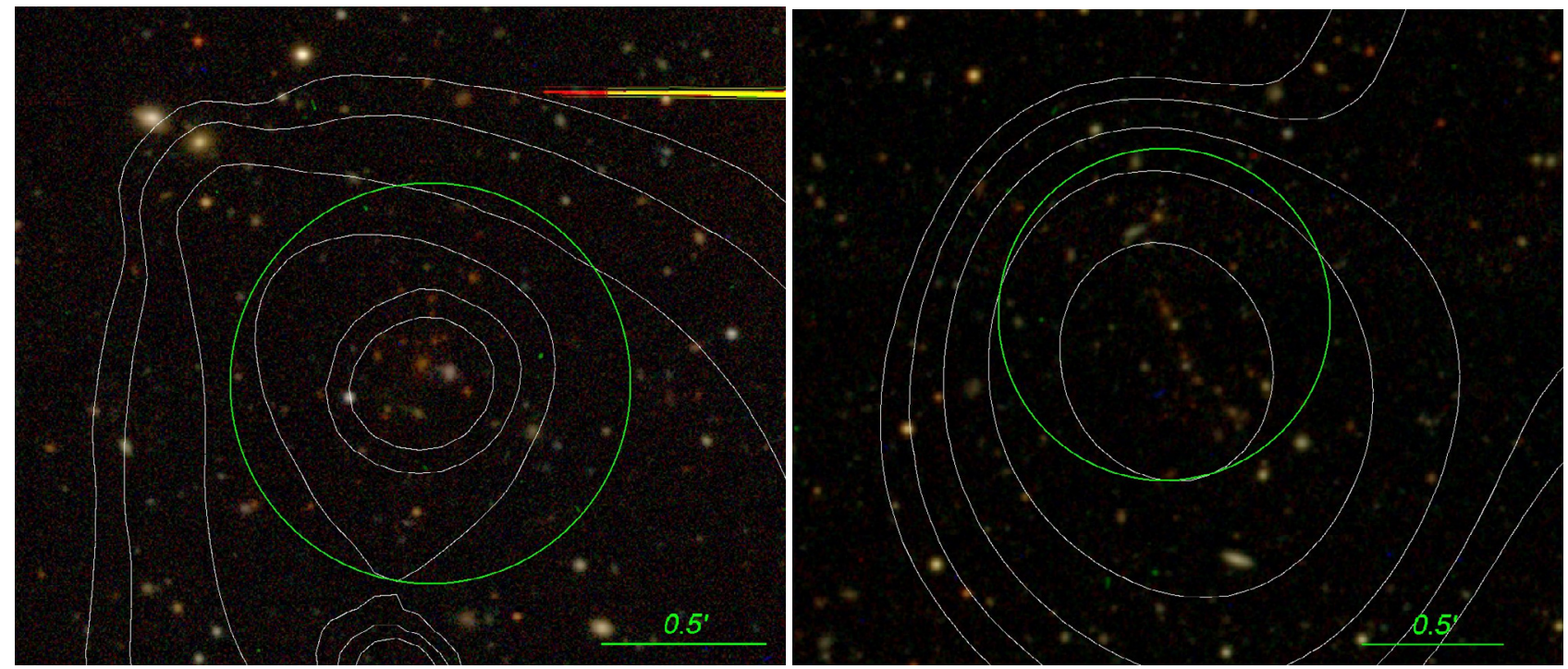

Fig. 7. Pseudo-color images in the gri bands of the two X-ray detected (white contours) systems with secure photo- $z$ values above $z>0.9$. Green circles mark the $0.5 \times r_{500}$ radius. Both clusters have a BCG coincident with the center of the $\mathrm{X}$-ray detection.

provide an important improvement in subsequent work. The final photometric redshifts are presented in Table 2. A more detailed analysis of optical counterparts for our systems including optical luminosity and richness estimates will be presented in a companion paper (Song et al., in prep.).

\subsection{Spectroscopic redshifts}

Spectroscopic redshifts are required to identify the clusters as compact objects, to derive precise physical parameters and later for cosmological modeling. In order to make a first step towards these goals we have carried out spectroscopic observations of a subsample of our clusters in the redshift range $z=0-0.4$.

The observations made use of the EFOSC2 instrument at the 3.6 m New Technology Telescope (NTT) in La Silla, Chile. The observations were carried out in September 2010, with typical exposure times of $840 \mathrm{~s}$ (two spectra per cluster, $420 \mathrm{~s}$ each). Our long-slit observations have been obtained using Grism \#4 (wavelength range 4085-7520 ). The slits (1.5" width) were placed on the BCG and an additional suitable cluster member candidate by rotating the slit. The BCG galaxies in these systems could be easily identified as the brightest red-sequence galaxies always coincident with the X-ray centroid, allowing us to safely anchor the cluster redshifts.

A standard reduction process was applied to the data using IRAF tasks ${ }^{4}$. The observations were bias subtracted, cleaned from cosmic rays, and flat fielded. For each galaxy we have obtained two spectra which were sky subtracted and combined

4 iraf.noao.edu 
Table 3. Spectroscopic redshift for 12 clusters in the redshift range $z=$ $0-0.4$.

\begin{tabular}{lcccc}
\hline \hline ID & $z_{\text {spec }}^{A}$ & $z_{\text {spec }}^{B}$ & $z_{\text {photo }}$ & $z_{\text {photo }}^{\text {SCS }}$ \\
\hline 70 & 0.152 & 0.152 & $0.17 \pm 0.03$ & 0.12 \\
94 & 0.269 & & $0.29 \pm 0.04$ & \\
127 & 0.207 & 0.209 & $0.22 \pm 0.02$ & \\
139 & 0.169 & & $0.18 \pm 0.01$ & \\
150 & 0.176 & 0.173 & $0.20 \pm 0.02$ & 0.14 \\
152 & 0.139 & & $0.17 \pm 0.02$ & \\
227 & 0.346 & & $0.35 \pm 0.04$ & \\
430 & 0.206 & 0.205 & $0.18 \pm 0.01$ & \\
457 & 0.1 & & $0.10 \pm 0.01$ & \\
476 & 0.102 & & $0.10 \pm 0.01$ & 0.1 \\
511 & 0.269 & & $0.26 \pm 0.02$ & 0.2 \\
547 & 0.241 & & $0.22 \pm 0.02$ & 0.18 \\
\hline
\end{tabular}

Notes. The redshifts were obtained from long-slit spectroscopic observations at the NTT telescope. The redshifts of the BCG galaxies are in the $z_{\text {spec }}^{A}$ column. Four clusters have a redshift for one additional member galaxy $\left(z_{\text {spec }}^{B}\right)$. Photometric redshifts $z_{\text {photo }}$ are taken from Table 2. For five systems we also provide the photometric redshifts from the SCS survey (M09, M10).

to increase the signal-to-noise ratio. The wavelength calibration was carried out by comparison with exposures of He and Ar lamps.

The final spectra were then correlated with a database of galaxy templates. In almost all cases the $\mathrm{H}$ and $\mathrm{K}$ lines and the $4000 \AA$ A break were visible and used for visual check of the template correction results. Spectroscopic redshifts have been secured for a total of 12 BCG galaxies. Due to relatively short exposures used only four systems a second member galaxy in the slit had good signal-to-noise ratio in order to safely measure its redshift. In all four cases the galaxies were found to have concordant redshifts with the BCG value. The spectroscopic redshifts of the galaxies are summarized in Table 3 along with our photo- $z$ estimates. We compare the two redshift sets in Sect. 4.2.

\subsection{Comparison of spectroscopic and photometric redshifts}

For a subsample of 12 clusters $(z<0.4)$ we have obtained spectroscopic redshifts of their BCG galaxy and in four systems also for one additional member galaxy (Sect. 4.1). We compare the spectroscopic redshifts with our photo- $z$ values in Fig. 8 (left). Our photometric values (red points) agree well within the error bars with the spectroscopic redshifts of the BCG (green points, brighter green points mark the clusters with two concordant redshifts). Blue points mark the photo-zs for five of the systems obtained by the SCS survey (M09, M10). These values exhibit a systematic bias toward lower redshifts, with a mean relative difference of $19 \%$. A similar trend is also visible in Fig. 13 (top left) where we compare our photo- $z$ values with the SCS measurements for all clusters common to both samples.

The right panel of Fig. 8, displays the comparison of the absolute difference of our photometric and spectroscopic estimates in units of photo- $z$ error, $D=\left|z_{\text {photo }}-z_{\text {spec }}\right| / \sigma_{\text {photo }}$. A comparison with a Gaussian expectation shows an agreement at the $96 \%$ confidence level, confirming both the good precision of our photo- $z$ estimates and the realistic description of their errors.

The present spectroscopic sample covers only part of the redshift range and does not allow us to check the photometric redshift calibration at higher redshift. However, the good agreement at low $z$ supports the photo- $z$ method used. We also note, that same photo- $z$ method has been applied to a large number of SPT selected clusters extending to beyond $z=1$, and the agreement between spectroscopic and photometric redshifts has been excellent (High et al. 2010; Williamson et al. 2011).

\section{Results}

\subsection{Galaxy cluster sample}

Table 2 provides the physical properties determined for the 46 clusters in the present sample. The measured X-ray luminosity of the systems (Sect. 3.3) and the photometric and spectroscopic redshifts (Sects. 4 and 4.1) are used as inputs for the cluster scaling relations to estimate further physical parameters. Ancillary X-ray information on the individual clusters can be found in Table A.1.

The redshift, temperature and mass distributions are shown in Fig. 9. We display the X-ray luminosity of our systems as a function of redshift in Fig. 10. The median redshift of the cluster sample is $z=0.47$. Six of the systems have photometric redshifts $z>0.8$. Three of these have redshifts consistent with $z=1$, although the photo- $z$ uncertainty in this regime is large. The median temperature of the clusters is $\sim 2 \mathrm{keV}$ and the median $M_{500}$ mass $9 \times 10^{13} M_{\odot}$ (based on luminosity scaling relations). We are thus able to probe the cluster/group transition regime practically at all redshifts out to $z \approx 1$.

\subsection{Survey sky-coverage}

The simplest statistical characteristics of a cluster survey are its area coverage as a function of limiting flux (sky-coverage function) and the cumulative surface density of the detected objects above the given flux limit as a function of flux - the so-called $\log N-\log S$ relation $^{5}$.

In order to properly determine the survey's sky coverage, good knowledge of the survey's selection function is necessary. For the simple case when the selection function is the function of only flux, the sky coverage is then the selection function of the survey scaled by its geometric area. Especially for the case of extended sources the situation is more complex, since the selection function depends also on other parameters (e.g. the source extent and off-axis angle). These effects can only be accounted for by Monte Carlo simulations. At this moment, without the simulations at hand, we can still provide a preliminary, empirically calibrated sky coverage calculation and cluster $\log N-\log S$ relation. We will demonstrate that these simple approaches show good agreement with the design aims for the survey depth and previous measurements of the cluster $\log N-\log S$ function.

While our source detection pipeline utilizes multiple detection bands and likelihood thresholds (Sect. 3) we will for simplicity (and ability to compare our results with published work) characterize detections made in the standard $0.5-2 \mathrm{keV}$ band with a $3 \sigma$ detection threshold and a $5 \sigma$ extent significance.

In order to obtain the survey sensitivity function for extended sources, we first calculate the point source sensitivity for each field. This is a simpler task since it does not require treatment of the source extent. We calculate the point source sensitivity function by analytically inverting the detection likelihood calculation (described in Sect. 3.1) and obtaining the minimal countrate necessary for a point source to be detected at the required

\footnotetext{
5 We use the standard notation of this relation, but keep writing $f_{\mathrm{X}}$ as the source flux rather than $S$.
} 

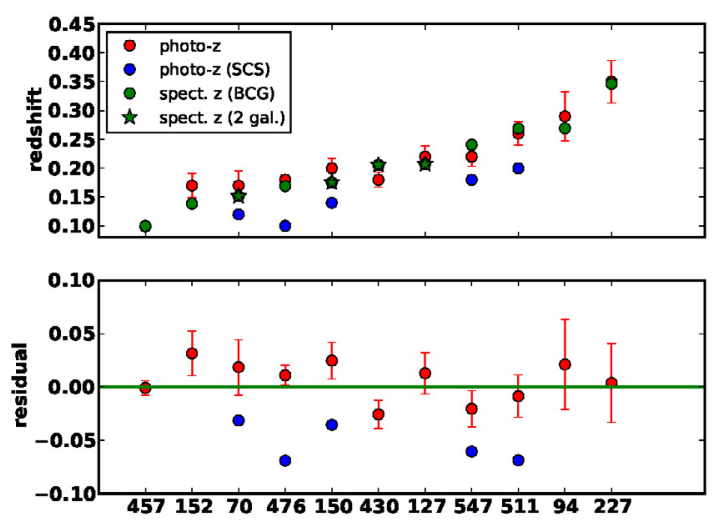

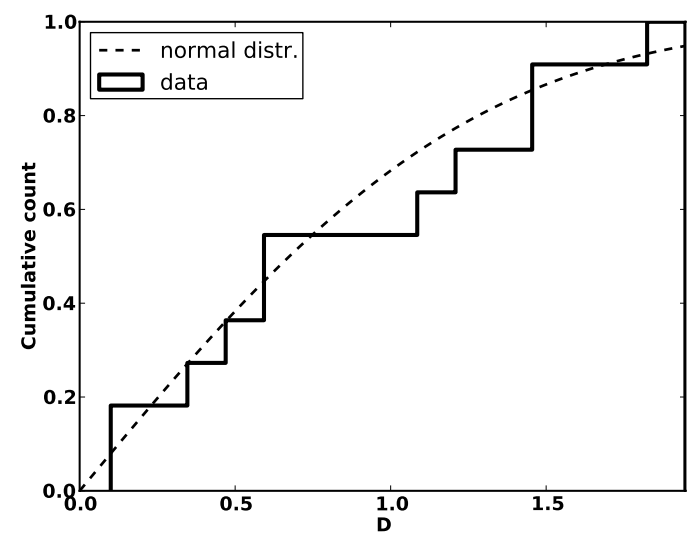

Fig. 8. Consistency test of our photometric redshift estimates with spectroscopic measurements in the redshift range $z=0-0.4$. Left: comparison of our photometric redshift estimates (red, $1 \sigma$ error bars) with spectroscopic values (green). Green stars mark clusters for which we have two concordant galaxy redshifts, while green circles indicate clusters for which only the BCG has a spectroscopic redshift. The photometric redshifts obtained by the SCS survey (M09, M10) are shown in blue. The $x$-axis displays the cluster ID number. The objects are sorted in increasing redshift order. The bottom panel shows the residuals of the photo- $z$ values with respect to the spectroscopic measurement. Right: cumulative histogram of the difference between the photometric and spectroscopic redshift normalized by the $1 \sigma$ uncertainty of the photo- $z$ values, i.e. $D=$ $\left|z_{\text {photo }}-z_{\text {spec }}\right| / \sigma_{\text {photo }}$. The dashed line shows the expectation for the Gaussian distribution. Both curves are in good agreement, with a KolmogorovSmirnov test confirming that the distribution of the $D$ values is Gaussian at the $96 \%$ confidence level.


Fig. 9. Top left: the redshift distribution of the 46 cluster sample based on the photometric redshifts obtained with the red sequence fitting method. Top right: the X-ray temperature distribution estimated from the L-T scaling relation of Pratt et al. (2009). Bottom: distribution of the cluster masses in the $r_{500}$ aperture calculated from the luminosities using the L-M scaling relation from Pratt et al. (2009, see Sect. 3.3).

detection threshold given the local background and exposure in the detection cell.

The procedure is repeated for each survey field and the results are combined for the whole survey area. In the areas where two or more fields overlap, we compare the sensitivity maps pixel-by-pixel taking the highest reached sensitivity (i.e. lowest local count-rate limit) at the given position. This procedure is chosen because the present catalog was derived from the detection pipeline that ran on each field individually. An alternative approach is to combine the fields before detection - reaching slightly deeper flux-limits in the overlapping areas ${ }^{6}$. This comes

${ }^{6}$ This was done for the ancillary catalog using the wavelet detection algorithm. 

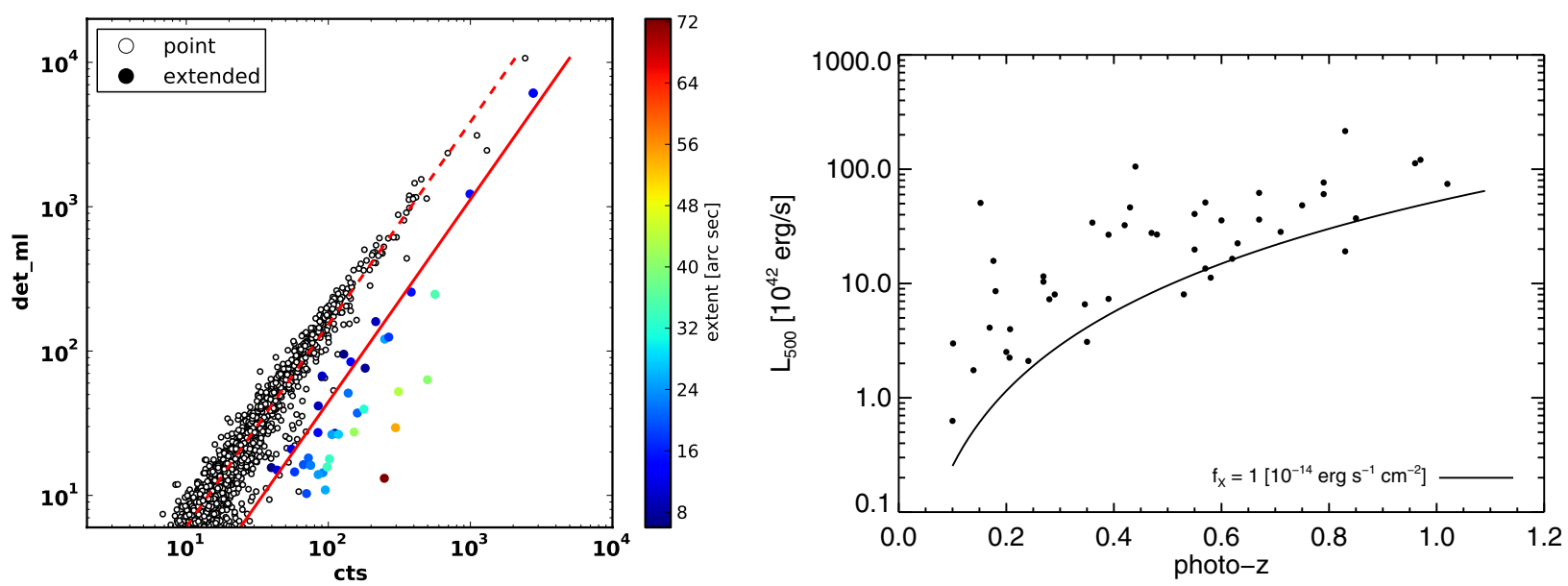

Fig. 10. Left: detection likelihood (det_ml) as a function of total detected source counts (PN detector only) for point sources (open circles) and the detected clusters (full circles). Clusters are color coded by their extent (beta model core radius). The dashed red line shows the best fit linear relation in the det_ml - counts plane for point sources. The solid line shows the same relation for extended sources (with slope fixed to the point-source fit, points weighted by their counts error). Typically, an extended source has to have 2.4 times more counts than a point source to be detected at the same det_ml value. See Sect. 5.2 for details. Right: luminosity in the $0.5-2 \mathrm{keV}$ band (object rest-frame) for the present cluster sample as a function of redshift. The line shows the luminosity of a cluster with a measured flux of $1 \times 10^{14} \mathrm{erg} \mathrm{s}^{-1} \mathrm{~cm}^{-2}$ (unabsorbed, observer rest-frame).

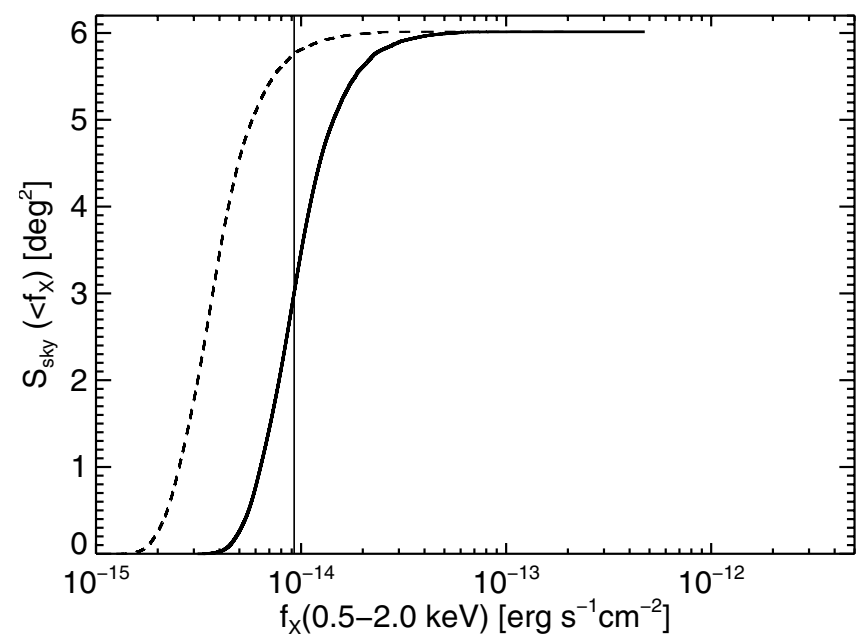

Fig. 11. The survey sky coverage. Dashed line shows the sky coverage as a function of limiting point source sensitivity in the $0.5-2.0 \mathrm{keV}$ band. The empirically estimated extended source sensitivity is shown with a solid line. The median point source sensitivity of the survey is $3.7 \times 10^{-15} \mathrm{erg} \mathrm{s}^{-1} \mathrm{~cm}^{-2}$, the median sensitivity for the extended sources $9.3 \times 10^{-15} \mathrm{erg} \mathrm{s}^{-1} \mathrm{~cm}^{-2}$ (vertical line).

at the cost of losing the information on the local PSF shape used by the maximum-likelihood fitting algorithm, since the same sky location in two different observations is imaged at different offaxis and position angles and thus with different PSF. Both approaches give comparable results and we opt here to characterize the main scheme (i.e. detection on individual fields).

The median point source sensitivity calculated in this way for the whole survey area is $3.7 \times 10^{-15} \mathrm{erg} \mathrm{s}^{-1} \mathrm{~cm}^{-2}$ for an energy-conversion factor ${ }^{7}$ of $1.5 \times 10^{-12} \mathrm{erg} \mathrm{s}^{-1} \mathrm{~cm}^{-2}$. The corresponding sky coverage as a function of flux is displayed in Fig. 11.

\footnotetext{
7 Assuming a power law spectrum with $\Gamma=1.7$ and $n_{\mathrm{H}}=1.25 \times$ $10^{20} \mathrm{~cm}^{-2}$ (median value of the galactic column density in the survey field) and using an on-axis PN response file.
}

In the next step, we attempt to obtain a first order approximation to the sky coverage function for the extended sources by a simple scaling to the point source function. In Fig. 10 (left) we show the dependence of the detection likelihood (i.e. the det_ml parameter) on the total detected source counts for point sources and the confirmed clusters from our sample (full circles).

Photons from extended sources are distributed over a larger area and thus require more counts to reach a given detection likelihood compared to point sources. For those, a simple linear relation in the log-log plane is a good description of the counts-det_ml relation (dashed red line in Fig. 10). Since the number of our clusters is small a similar linear relation for them is only very weakly constrained. We therefore fix the slope to the value from the point source fit leaving only the intercept as a free parameter and weighting the points by their counts error (solid red line). The offset of the extended-source best-fit line translates to a factor of 2.4 between the total required counts of point and extended sources at any given det_ml. Fixing the slope has also the advantage that the offset is independent of the selected detection threshold. The best fit line roughly follows the locus of clusters with extent (beta model core radius) close to the median value of $\sim 20^{\prime \prime}$. The solid red line in Fig. 10 (left) thus roughly gives the detected counts for a cluster with a typical extent detected with a given likelihood. We then use this offset factor to scale up the point source sky coverage function (see Fig. 11). The median flux limit for this sky-coverage is $9.3 \times 10^{-15} \mathrm{erg} \mathrm{s}^{-1} \mathrm{~cm}^{-2}$ (using the median ECF of our sample). In Fig. 10 we display the luminosity-redshift plane for our survey. The luminosity threshold for a flux limited sample $\left(f_{\min }=1 \times 10^{-14} \mathrm{erg} \mathrm{s}^{-1} \mathrm{~cm}^{-2}\right)$ is also shown, demonstrating a rough agreement with our calculation. Note that in the present sample we also include fainter sources than this threshold (the lowest cluster flux is $\sim 6 \times 10^{-15} \mathrm{erg} \mathrm{s}^{-1} \mathrm{~cm}^{-2}$ ).

This approach underestimates the effect of clusters with larger extent - and thus overestimates the sky coverage at given flux ${ }^{8}$. However, since the detection probability itself is a strong

\footnotetext{
8 The fit with a free slope gives an offset factor of $\sim 4$, the fit being skewed towards the locus of very extended clusters.
} 
function of source extent, the only way to properly account for its effect is through realistic simulations.

We present examples of preliminary sky coverage functions for extended source detection on several (non-XMM-BCS) fields based on such Monte Carlo simulations (Mühlegger 2010) in Fig. B.1, discussed in Sect. B. These first results validate our attempt to model to first approximation the extended source skycoverage by scaling the point source curve and also confirm that the scaling factor between them is roughly $\sim 2.4$ (this scaling factor is expected to hold only for observations with roughly same depth as ours, $\sim 10 \mathrm{ks}$ ).

\subsection{Cluster $\log N-\log S$}

We now use this empirical sky-coverage in order to calculate the survey's $\log N-\log S$, defined in a standard way as:

$N\left(>f_{\mathrm{X}}\right)=\sum_{i=1}^{N_{\mathrm{C}}} \frac{1}{\Omega\left(f_{\mathrm{X}}^{i}\right)} \mathrm{deg}^{-2}$,

where $N_{\mathrm{C}}$ is the total number of clusters and $\Omega\left(f_{\mathrm{X}}^{i}\right)$ is the extended source sky-coverage corresponding to the flux of the $i$ th cluster. We characterized the survey sky coverage only for a hypothetical single band ( $0.5-2 \mathrm{keV})$ detection scheme. Since such a detection scheme is not part of our pipeline, we opt to draw a subsample from our cluster catalog derived from the three band scheme (which includes the $0.5-2 \mathrm{keV}$ ). For this calculation we consider only clusters that would have also been detected in this hypothetical single band run by setting the same detection- and extent likelihood thresholds used for the sky-coverage calculation in the previous section.

This requires us to recover the actual $0.5-2 \mathrm{keV}$ band detection likelihoods from the total det_ml parameter, which includes contributions from all three detection bands. As we described in Sect. 3, det_ml can be interpreted as det_ml = $-\ln P_{\text {rand }}$, with $P_{\text {rand }}$ being the probability of a false detection arising from pure Poissonian fluctuations. The actual definition of this parameter is slightly more complex:

det_ml $=-\ln (1-\Gamma(0.5 v, L))$,

where $\Gamma$ is the incomplete gamma function and its arguments are the number of degrees of freedom of the emldetect fit (for extended sources $v=3+$ the number of detection bands times number of instruments) and $\mathrm{L}$ is the sum of all the individual likelihoods (using the $C$ statistics of Cash 1979). This definition effectively converts the joint likelihoods to two degrees of freedom allowing to compare detections from different combination of bands and instruments. However, for the conversion to a single band detection likelihood, we need the original individual likelihoods which we obtain by numerically inverting Eq. (6) for each source.

We then calculate the joint detection likelihood from all three instruments in the single, $0.5-2 \mathrm{keV}$, band (all three detection probabilities being independent) and subsequently calculate the new det_ml parameter normalized back to two degrees of freedom using Eq. (6). The number of clusters that have this new single band det_ml parameter above the required threshold (i.e. equivalent to $\sim 3 \sigma$ ) is 40 .

Finally, we calculate the $\log N-\log S$ according to Eq. (5) and the variance of the number counts as $\sigma^{2}=\sum_{i=1}^{N_{\mathrm{C}}} 1 / \Omega\left(f_{\mathrm{X}}^{i}\right)^{2}$. The recovered curve (see Fig. 12) is in good agreement with the $\log N-\log S$ of other surveys: e.g. COSMOS (Finoguenov et al. 2007), the RDCS survey (the ROSAT Deep Cluster Survey,

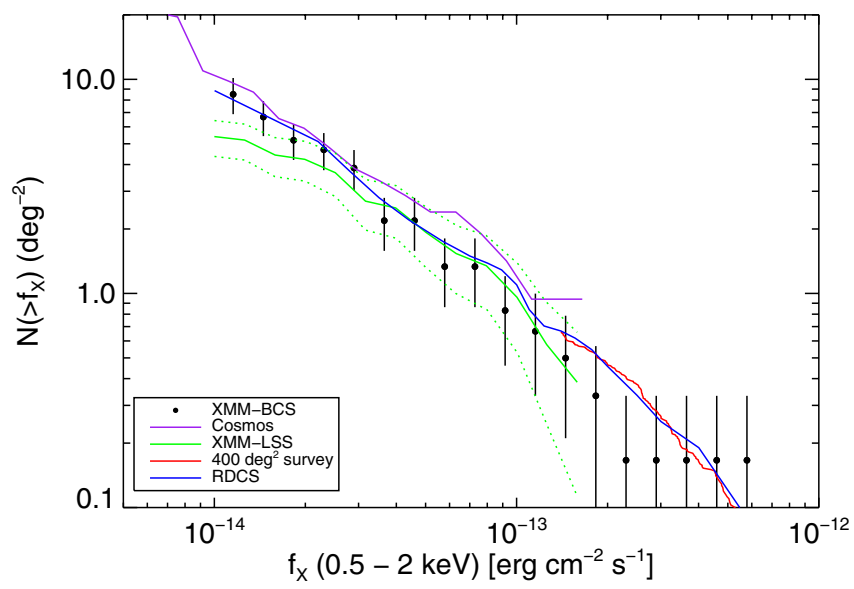

Fig. 12. The $\log N-\log S$ of the present sample in the $0.5-2.0 \mathrm{keV}$ band. Fluxes are calculated in the $r_{500}$ aperture. Results from several surveys are also shown: COSMOS (Finoguenov et al. 2007), RDCS (the ROSAT Deep Cluster Survey, Rosati et al. 1998), $400 \mathrm{deg}^{2}$ survey (Burenin et al. 2007; Vikhlinin et al. 2009) and the XMM-LSS (Pacaud et al. 2007). See Sect. 5.3 for details.

Rosati et al. 1998), $400 \mathrm{deg}^{2}$ survey (Burenin et al. 2007; Vikhlinin et al. 2009) and the XMM-LSS survey ${ }^{9}$ (Pacaud et al. 2007). Since the area and depth of the XMM-LSS survey match well the parameters of our survey we discuss their comparison in more detail in Sect. C.1.

We note, that we used the fluxes in the $r_{500}$ aperture for our calculation whereas the XMM-LSS uses a fixed physical aperture of $0.5 \mathrm{Mpc}$ (typically very close to $r_{500}$ ), RDCS $\sim 80-90 \%$ of the total flux (i.e. integrated out to infinity) and the $400 \mathrm{deg}^{2}$ survey the full total flux. We have chosen $r_{500}$ because: a) it requires less extrapolation based on a beta model whose parameters are typically highly uncertain and is itself not necessarily a good description of the surface brightness profiles and $b$ ) it is the most natural choice when comparing to theoretical predictions (the cluster is approximately virialized in this radius and the scaling relations we employ are calibrated for this overdensity). However, assuming a typical cluster with $r_{500}=0.5 \mathrm{Mpc}$ well described by a beta model with $\left(\beta, r_{\text {core }}\right)=(2 / 3,180 \mathrm{kpc})$ the flux extrapolated to infinity would be higher by $\sim 1 / 3$ moving our curve along the $x$-axis to higher fluxes only very slightly - even closer to the RDCS and $400 \mathrm{deg}^{2}$ survey's relations.

Uncertainties of the flux estimation (including the uncertainty on the photo- $z s)$ affect the $\log N-\log S$ only in a minor way. The main source of uncertainty (not included in the error bars) is our current lack of knowledge of the survey selection function (and thus only tentative description of the sky-coverage). The good agreement with previous work gives, however, support to our preliminary approach.

\subsection{Cross-correlation with known sources}

The XMM-BCS field has an extensive multi-wavelength coverage and has already been studied by the Southern Cosmology Survey (M09; M10; McInnes et al. 2009) who identified in optical data a number of clusters in this area. Due to a significant overlap with our cluster catalog we will address a more detailed comparison in Sect. 5.5.

${ }^{9}$ Note that the XMM-LSS curve is only digitized from the figure in Pacaud et al. (2007) since the original curve is no longer available (Pacaud, priv. comm.). 
R. Šuhada et al.: The XMM-BCS galaxy cluster survey. I.

Table 4. Radio sources within $60^{\prime \prime}$ from the X-ray centers of the detected clusters.

\begin{tabular}{cccccc}
\hline \hline ID & Object name & RA $(\mathrm{deg})$ & Dec $(\mathrm{deg})$ & $S(\mathrm{mJy})$ & Separation $\left({ }^{\prime \prime}\right)$ \\
\hline 018 & SUMSS J232952-560723 & 352.4677 & -56.1231 & $14.9 \pm 0.8$ & 56 \\
035 & SUMSS J233345-553817 & 353.4416 & -55.6382 & $16.7 \pm 0.8$ & 6 \\
044 & SUMSS J231654-545406 & 349.2274 & -54.9019 & $8.0 \pm 1.0$ & 14 \\
109 & SUMSS J232737-541622 & 351.9047 & -54.2730 & $26.1 \pm 1.2$ & 9 \\
110 & SUMSS J233003-541424 & 352.5146 & -54.2402 & $13.3 \pm 1.1$ & 6 \\
189 & SUMSS J233044-560123 & 352.6860 & -56.0233 & $15.1 \pm 0.8$ & 36 \\
210 & SUMSS J233406-554708 & 353.5253 & -55.7857 & $7.9 \pm 0.7$ & 3 \\
288 & SUMSS J233459-545535 & 353.7495 & -54.9265 & $41.5 \pm 1.6$ & 37 \\
426 & SUMSS J232138-541849 & 350.4092 & -54.3137 & $12.7 \pm 1.9$ & 14 \\
534 & SUMSS J232446-552432 & 351.1951 & -55.4089 & $41.1 \pm 1.5$ & 17 \\
546 & SUMSS J233113-543025 & 352.8076 & -54.5071 & $25.6 \pm 1.3$ & 26 \\
150 & PMN J2330-5436 & 352.5075 & -54.6097 & $52.0 \pm 8.0$ & 34 \\
\hline
\end{tabular}

Notes. The quoted flux density $\mathrm{S}$ is at $843 \mathrm{Mhz}(36 \mathrm{~cm})$ for the SUMSS sources and at $4.85 \mathrm{GHz}(6.2 \mathrm{~cm})$ for the PMN detected object. The radio counterparts were obtained from the NASA/IPAC Extragalactic Database.

In search of other known sources coincident with our clusters, we make use of both the NASA/IPAC Extragalactic Database $^{10}$ and the SIMBAD Astronomical Database ${ }^{11}$.

First we looked for associated known clusters. For this query a search radius of $60^{\prime \prime}$ was selected, finding a single match the cluster 400d J2325-5443 (alternative name: [BVH2007] 240) identified in the 160 Square Degree ROSAT Survey (Vikhlinin et al. 1998; Mullis et al. 2003) at spectroscopic redshift $z=$ 0.102 . This cluster is coincident with our cluster ID 476 with photometric redshift of 0.1 being in full agreement with the spectroscopic value. The source is also part of the 400 Square Degree ROSAT Survey. See Appendix A.2 for more details on this source.

We also list galaxy matches, if they are within a 16 arcsec search radius from the X-ray center in Table A.2 with matches coming from the 2 Micron All Sky Survey Extended objects catalog and the APM galaxy survey (Skrutskie et al. 2006; Maddox et al. 1990, respectively). Out of 13 matches, only two galaxies have known spectroscopic redshifts, both obtained in the frame of the 6dF Galaxy Survey (Jones et al. 2004). The first is 2MASX J23254015-5444308 at $z=0.101$ coincident with the brightest galaxy in cluster ID 476 . The redshift value is concordant with the redshift from the 160/400 Square Degree ROSAT surveys. The second match is the brightest cluster galaxy of the system ID 150 at redshift $z=0.176$, again in good agreement with our estimated photo- $z$ of 0.2 .

As can be seen, the survey field has a wealth of multiwavelength data, but very little spectroscopic measurements. This makes the ongoing spectroscopic follow-up program very important, as redshifts are essential for the full utilization of the available data sets.

Radio sources coincident with the X-ray detected clusters can bias the SZE signal (filling the decrement). We checked for intervening radio sources by cross-correlating our cluster catalog with the NED database with a 1 arcmin search radius. We find 11 radio sources detected at $843 \mathrm{MHz}$ by the Sydney University Molonglo Sky Survey (SUMS, Mauch et al. 2003). The source PMN J2330-5436, 30 arcsec from cluster ID 150, was detected by the Parkes-MIT-NRAO (PMN) southern survey at $4.85 \mathrm{GHz}$ (Gregory et al. 1994). The list of all identified radio sources is given in Table 4.

\footnotetext{
10 nedwww.ipac.caltech. edu

11 simbad.u-strasbg.fr/simbad/
}

\subsection{Cross-correlation with the Southern Cosmology Survey clusters}

The Southern Cosmology Survey (SCS) carried out an optical cluster search using the Blanco Cosmology Survey imaging data. Menanteau et al. (2009, hereafter M09) provided a catalog of optically selected clusters with photo- $z<0.8$ in a 8 deg $^{2}$ field partially overlapping with the $6 \mathrm{deg}^{2}$ region presented in this work. Menanteau et al. (2010, M10) then followed up this work by creating a cluster catalog using the full 2005-2007 BCS survey data (i.e. $\sim 70 \mathrm{deg}^{2}$, thus fully covering also the whole XMMBCS field), detecting 105 clusters with $M_{200}>3 \times 10^{14} M_{\odot}$ and photo- $z<0.8$.

Combining both these catalogs, we find in total 30 SCS clusters whose optical coordinates lie inside our $6 \mathrm{deg}^{2}$ region $^{12}$. Out of these 30 systems, 26 come from the M09 catalog (which contains clusters also below the mass limit applied in M10), two are included in both M09 and M10 and two additional clusters are from M10. For the two clusters in both M09 and M10 we will use the updated parameters from M10.

The SCS catalog provides the BCG coordinates for each system while our catalog lists the X-ray centroids. For crosscorrelation of the two catalogs we take a conservative $60^{\prime \prime}$ matching radius, which yields 19 clusters. We summarize the properties of matched clusters in Table 5 and provide a more detailed comparison of their parameters in the next two sections.

\subsubsection{Comparison of photometric redshifts}

First, we turn to the comparison of the photometric redshifts for the 19 matches. M09 and M10 utilize the BPZ code (Benítez $2000)$ to estimate photo- $z s$ while our method is based on the redsequence method as described in Sect. 4. For the SCS clusters we use the photo- $z$ errors published in McInnes et al. (2009) where possible (the M09 and M10 catalogs do not provide error bars). For the remaining cases we assume a $15 \%$ error, which is the mean precision of the photo- $z s$ where errors are available.

As can be seen in Fig. 13 (top left) there is no case of catastrophic disagreement. We find a gap in the SCS photo- $z$ distribution in the $0.35-0.5$ photo- $z$ range that is not present in our redshift distribution. The most important feature is, however, the systematic offset between the photo- $z$ estimates. The SCS photo-zs are on average $\sim 20 \%$ lower than our values. This trend

\footnotetext{
12 In the present work we thus do not consider SCS clusters that lie only partially in our $6 \mathrm{deg}^{2}$ region or in the $8 \mathrm{deg}^{2}$ mosaic extension.
} 

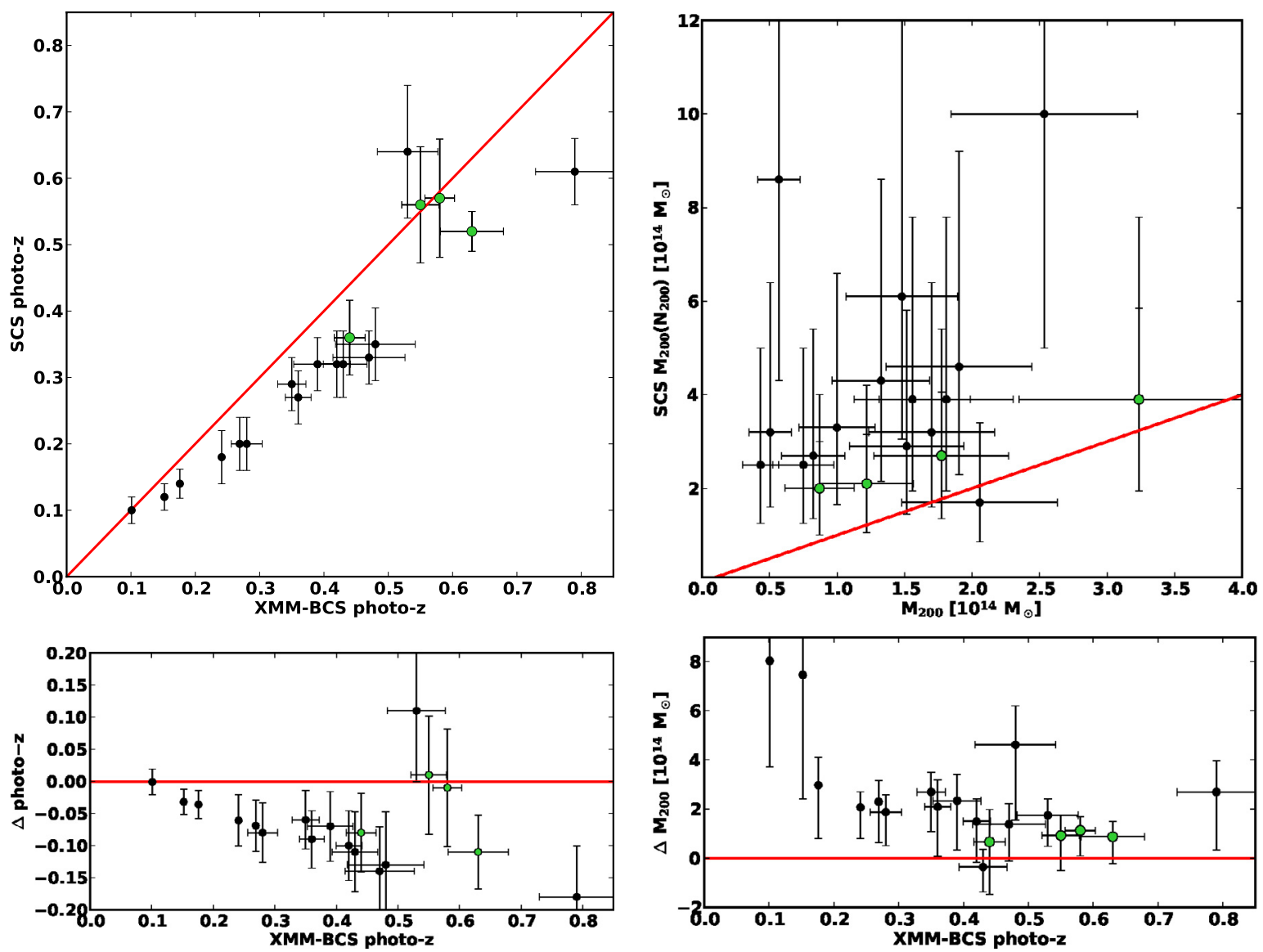

Fig. 13. Top left: comparison of photometric redshifts for the 19 common clusters from our sample and the SCS cluster survey from Menanteau et al. (2009) and Menanteau et al. (2010). Top right: comparison of masses for the same cluster sample in the $r_{200}$ aperture determined from the measured X-ray luminosity through scaling relations ( $x$-axis) and the mean of the optically determined masses $M\left(L_{200}^{\mathrm{opt}}\right)$ and $M\left(N_{200}\right)($ Table 5). The red line marks equality in both top panels. Bottom left: photo- $z$ difference $\Delta=$ photo- $z$ (SCS) - photo- $z$ (XMM-BCS) as a function of our estimates of redshifts. Bottom right: $M_{200}$ difference $\Delta=M_{200}$ (SCS) $-M_{200}$ (XMM-BCS) as a function of our estimates of redshifts. The optical masses are significantly higher than the X-ray estimates especially at the low and high redshift ends. See text for discussion. Green points in all plots mark clusters from M10, black points those from M09.

roughly holds in the whole redshift range, as can be seen from the photo- $z$ residuals plotted against redshift in Fig. 13, bottom left). We found a similar bias when comparing the SCS photo- $z s$ to the spectroscopic subsample in Sect. 4.2. For the five systems with spectroscopic redshifts the photo- $z s$ were on average underestimated by $\sim 19 \%$.

In order to investigate potential sources of the discrepancy we check whether the photo- $z$ residuals depend on any of the available parameters, most importantly the richness parameter $N_{200}$, integrated optical luminosity $L_{200}^{\mathrm{opt}}$ and the BCG-X-ray centroid offset. However, we do not find any statistically significant dependence.

\subsubsection{X-ray - optical mass comparison}

M09 and M10 provide rough mass estimates for their clusters based on the optical proxies $N_{200}$ and $L_{200}^{\text {opt }}$ using the scaling relations from Reyes et al. (2008). The richness estimator $N_{200}$ is defined as the number of $\mathrm{E} / \mathrm{S} 0$ ridgeline cluster members brighter than $0.4 L^{*}$. The integrated cluster luminosity $L_{200}^{\mathrm{opt}}$ is the summed $r$ band luminosity of the member galaxies included in the $N_{200}$ calculation. Both parameters are calculated within an aperture where the galaxy density equals $200 / \Omega_{M}$ times the mean density of galaxies in the Universe. Fortunately, Johnston et al. (2007) found that this aperture is an unbiased estimate of the radius where the matter density is 200 times the critical density of the Universe, i.e. the optical masses and our X-ray estimates come from roughly the same apertures and can thus be directly compared.

In Fig. 13 (top right) we compare our X-ray masses with the optical masses $M\left(N_{200}\right)$ calculated from the $N_{200}$ parameter. The optical masses are estimated to be accurate within a factor of two (M09), where this factor should include also the uncertainty in extrapolating the Reyes et al. (2008) scaling relations to higher redshifts (the scaling relations were calibrated for redshifts $z<0.3)$. We used the factor two uncertainty to calculate the $M\left(N_{200}\right)$ error bars in Fig. 13. We find that the optical masses are significantly higher than the X-ray mass estimates $M_{200}$ by a factor of $\sim 2.6$ (median value).

Reichert et al. (2011) investigate X-ray luminosity based scaling relations on a large compilation of cluster samples from the literature. They find only very few systems deviating from the mean $L-M$ relation by more than a factor two (i.e. with actual mass two or more times higher than the luminosity prediction). We thus do not expect our X-ray masses to be underestimated by similar factors even in individual cases. The observed bias in mass goes in the opposite direction as that found in the photo-zs (i.e. photo-zs were underestimated while masses overestimated). The photo- $z s$, however influence the mass estimates 
R. Šuhada et al.: The XMM-BCS galaxy cluster survey. I.

Table 5. The 19 SCS clusters recovered in the XMM-BCS survey.

\begin{tabular}{|c|c|c|c|c|c|c|c|c|}
\hline SCS ID & Ref. & Photo-z (SCS) & $M\left(L_{200}^{\mathrm{opt}}\right)$ & $M\left(N_{200}\right)$ & $M_{200}^{\mathrm{wl}}$ & $M_{200}^{\mathrm{X}}$ & XMM-BCS ID & Separation (") \\
\hline SCSO J233430.2-543647.5 & M09 & 0.35 & 36 & 61 & - & $14.8 \pm 4.1$ & 357 & 26.6 \\
\hline SCSO J232211.0-561847.4 & M09 & 0.61 & 56 & 46 & $4.7_{-4.7}^{+26.1}$ & $19.0 \pm 5.4$ & 527 & 1.6 \\
\hline SCSO J232540.2-544430.9 & M09 & 0.10 & 21 & 86 & $2.3_{-3.3}^{+8.9}$ & $5.7 \pm 1.6$ & 476 & 3.2 \\
\hline SCSO J232230.9-541608.3 & M09 & 0.12 & 16 & 100 & $8.5_{-5.9}^{5.9}$ & $25.4 \pm 6.9$ & 070 & 0.6 \\
\hline SCSO J233000.4-543707.7 & M09 & 0.14 & 12 & 43 & - & $13.3 \pm 3.6$ & 150 & 1.7 \\
\hline SCSO J232419.6-552548.9 & M09 & 0.18 & 12 & 25 & $<2.6$ & $4.3 \pm 1.3$ & 547 & 1.0 \\
\hline SCSO J233215.5-544211.6 & M09 & 0.20 & 17 & 33 & $10.2_{-61}^{+8.4}$ & $10.0 \pm 2.8$ & 511 & 10.0 \\
\hline SCSO J233037.1-554338.8 & M09 & 0.20 & 10 & 27 & $16.2_{-77}^{+10.7}$ & $8.2 \pm 2.3$ & 034 & 2.3 \\
\hline SCSO J232200.4-544459.7 & M09 & 0.27 & 17 & 39 & $2.6_{-26}^{+8.6}$ & $18.1 \pm 5.0$ & 136 & 3.9 \\
\hline SCSO J233522.6-553237.0 & M09 & 0.29 & 22 & 32 & $8.5_{-85}^{+16.0}$ & $5.1 \pm 1.6$ & 528 & 17.4 \\
\hline SCSO J232956.0-560808.3 & M09 & 0.32 & 20 & 39 & $21.3_{-17 .}^{+27.5}$ & $15.6 \pm 4.3$ & 018 & 1.7 \\
\hline SCSO J232839.5-551353.8 & M09 & 0.32 & 10 & 17 & $16.9_{-132}^{+20.1}$ & $20.6 \pm 5.8$ & 088 & 36.2 \\
\hline SCSO J232633.6-550111.5 & M09 & 0.32 & 28 & 32 & $<4.8$ & $17.0 \pm 4.7$ & 126 & 3.1 \\
\hline SCSO J233003.6-541426.7 & M09 & 0.33 & 9 & 29 & $28.1_{-147}^{+20.7}$ & $15.2 \pm 4.2$ & 110 & 7.4 \\
\hline SCSO J232619.8-552308.8 & M09 & 0.52 & 12 & 21 & $28.1_{-22.2}^{+33.4}$ & $12.2 \pm 3.5$ & 082 & 9.5 \\
\hline SCSO J231651.0-545356.0 & M10 & 0.36 & 27 & 39 & - & $32.4 \pm 8.9$ & 044 & 24.7 \\
\hline SCSO J232856.0-552428.0 & M10 & 0.57 & 35 & 20 & - & $8.7 \pm 2.6$ & 090 & 6.7 \\
\hline SCSO J233420.0-542732.0 & M10 & 0.56 & 36 & 27 & - & $17.7 \pm 5.0$ & 158 & 41.6 \\
\hline SCSO J233556.0-560602.0 & M10 & 0.64 & 47 & 25 & - & $7.5 \pm 2.3$ & 386 & 31.3 \\
\hline
\end{tabular}

Notes. SCS References: M09 - Menanteau et al. (2009), M10 - Menanteau et al. (2010). The optical masses $M\left(L_{200}^{\mathrm{opt}}\right)$ and $M\left(N_{200}\right)$ are taken from M09 and M10, the weak lensing mass measurements are provided by McInnes et al. (2009). The X-ray mass estimates obtained in the present work are taken from Table 2. The ID of the X-ray counterpart and its distance from the BCG are listed in the last two columns. The masses are in units of $10^{13} M_{\odot}$.

and therefore it is not straightforward to disentangle all the factors contributing to this discrepancy. The influence of the redshift uncertainty is likely more important for nearby systems, where it translates to larger differences in the angular size of the aperture. The discrepancy for the $M\left(L_{200}^{\mathrm{opt}}\right)$ masses is similar. We note here, however, that the $M\left(L_{200}^{\mathrm{opt}}\right)$ masses in M09 were obtained from the scaling relations of Reyes et al. (2008) prior to their erratum-correction ${ }^{13}$.

The bottom right panel of Fig. 13 displays the mass residuals versus our photometric redshifts. We find an anticorrelation between redshift and the mass residuals (using a Spearman's rank correlation coefficient, neglecting the error bars). Given that the Reyes et al. (2008) relations are calibrated only out to $z \lessgtr 0.3$ it is however impossible to either confirm the presence of such an anticorrelation or its potential causes. We also note that the four clusters from M10 agree with our measurements better than all but one cluster from M09.

McInnes et al. (2009) provides weak lensing mass measurements for the clusters from M09 among which 13 clusters are also in our sample (for two of these systems only upper limits could be set). We compare the weak lensing masses with our $\mathrm{X}$-ray estimates in Fig. 14. The agreement is significantly better than for $M\left(N_{200}\right)$ masses, although the scatter and uncertainty in the weak lensing mass estimates are large (mostly due to the limited depth of the optical data, but also influenced by the uncertainty in the photo- $z s$ ). From their full sample, McInnes et al. (2009) also noted that the $M\left(L_{200}^{\mathrm{opt}}\right)$ seems to overestimate the total mass compared to the weak lensing estimates.

An in-depth comparison of optical and X-ray masses will be addressed in an upcoming work, where we will provide also our own measurements of $N_{200}$ and $L_{200}^{\text {opt }}$ (Song et al., in prep.). This will allow us to properly investigate the presence of potential

\footnotetext{
13 Scaling relations with the updated coefficients are available at: arxiv.org/abs/0802.2365.
}

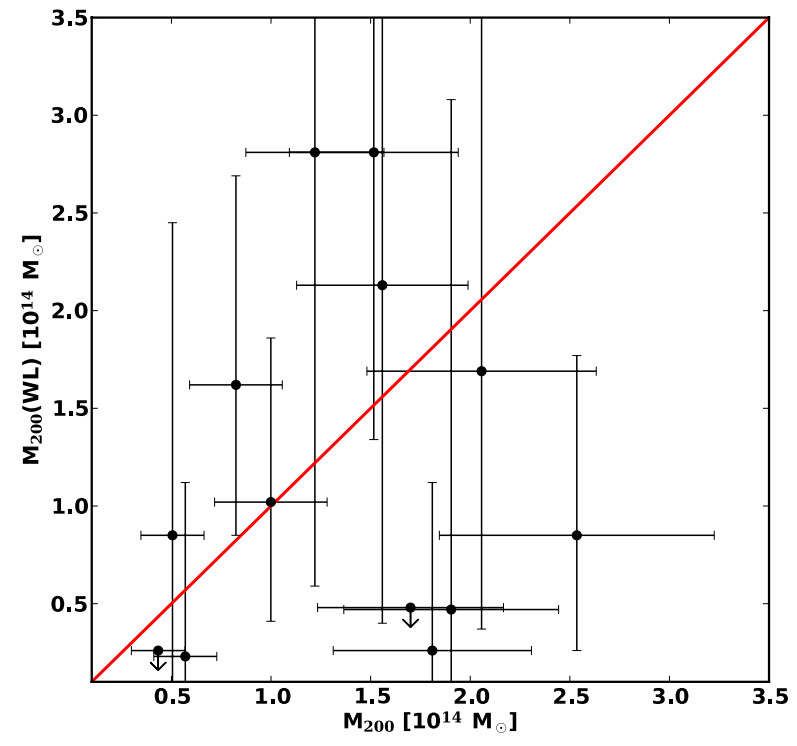

Fig. 14. Comparison of X-ray masses $\left(M_{200}, x\right.$-axis) with the weak lensing measurements (M(WL), $y$-axis) from McInnes et al. (2009) for 13 clusters in our sample. Although the scatter and uncertainties are large, the agreement is considerably better than with the optical masses $M\left(N_{200}\right)$ displayed in Fig. 13.

biases in the different mass estimators methods and calibrate our own relations.

\subsubsection{Parameter upper limits for $\mathrm{X}$-ray non-detections}

For the 11 SCS clusters that lie in the core area of our survey but have no X-ray counterparts we provide X-ray flux upper limits in Table 6 and mass limits using the SCS photo- $z$ values.

The flux limits were calculated using the same procedure as we used for the survey sky coverage calculation (Sect. 5.2, i.e. 
Table 6. The 11 SCS clusters from Menanteau et al. (2009) that lie in the XMM-BCS core survey but have no X-ray cluster detection, for which we provide flux and mass upper limits.

\begin{tabular}{lcccc}
\hline \hline SCS ID & $\begin{array}{c}\text { Photo- } z \\
(\mathrm{SCS})\end{array}$ & $\begin{array}{c}f_{\mathrm{X}}^{\lim } \\
\left(10^{-14} \mathrm{erg} \mathrm{s}^{-1} \mathrm{~cm}^{-2}\right)\end{array}$ & $\begin{array}{c}L_{500}^{\lim } \\
\left(10^{43} \mathrm{erg} \mathrm{s}^{-1}\right)\end{array}$ & $\begin{array}{c}M_{500}^{\lim } \\
\left(10^{13} M_{\odot}\right)\end{array}$ \\
\hline SCSO J232829.7-544255.4 & 0.68 & 0.79 & 1.6 & 5.5 \\
SCSO J233106.9-555119.5 & 0.19 & 0.69 & 0.1 & 1.3 \\
SCSO J233550.6-552820.4 & 0.22 & 0.68 & 0.1 & 1.6 \\
SCSO J232156.4-541428.8 & 0.33 & 0.88 & 0.3 & 2.8 \\
SCSO J233231.4-540135.8 & 0.33 & 1.59 & 0.6 & 4.0 \\
SCSO J233110.6-555213.5 & 0.39 & 0.73 & 0.4 & 3.1 \\
SCSO J233618.3-555440.3 & 0.49 & 1.51 & 1.4 & 5.9 \\
SCSO J232215.9-555045.6 & 0.56 & 0.84 & 1.1 & 4.8 \\
SCSO J232247.6-541110.1 & 0.57 & 0.72 & 0.9 & 4.4 \\
SCSO J232342.3-551915.1 & 0.67 & 1.22 & 2.4 & 7.0 \\
SCSO J233403.7-555250.7 & 0.71 & 0.56 & 1.3 & 4.7 \\
\hline
\end{tabular}

calculating the minimal flux needed for the source to be detected at the given position and our detection threshold).

The flux was then converted to luminosity using the photometric redshift from either M09 or M10. We calculated the mass upper limits from the $L-M$ scaling relation as detailed in Sect. 3.3. The obtained upper limits on the mass are considerably lower than the M09/M10 estimates.

We also check for possible miss-classification (or confusion if a central AGN is present) by cross-correlating the positions of these 11 clusters with our X-ray point source catalog with a threshold of $16^{\prime \prime}$. In this aperture we find no matches. The nondetection of these sources is due to either their low X-ray fluxes or a spurious detection.

\section{Discussion}

In this section we discuss the additional effects that influence the precision of the physical parameters provided in our catalog. We also give an outlook on the upcoming work in the context of the XMM-BCS survey.

\subsection{Error budget of the $X$-ray analysis}

For the present catalog, we restricted ourselves to provide only formal statistical errors for the estimated parameters (Table 2) that include the Poisson errors of the flux measurement, a $5 \%$ systematic error from the background modeling and the intrinsic scatter of the scaling relations. Although we used the bolometric luminosity to calculate further physical parameters, here we assumed the intrinsic scatter found in the $0.5-2 \mathrm{keV}$ luminosity relations. This scatter is slightly larger than the bolometric one and it gives a more realistic error estimate since the band luminosity is, in fact, our only direct observable, while the temperature required for the bolometric correction is not. We determine physical parameters with the following precision (mean across the whole redshift and flux ranges): flux and luminosity to $\sim 16 \%, T_{500}$ and $M_{500}$ to $\sim 30 \%$, and $Y_{500}$ to $\sim 60 \%$.

In this section we discuss several additional sources of systematic errors and their impact on the estimated fluxes and other parameters. All below reported relative errors are obtained by averaging over the whole cluster sample. Several of the considered effects are redshift dependent, but we typically allow broad parameter ranges and thus our uncertainty estimates are rather conservative.

1. Good precision photometric redshifts are crucial for the determination of each physical parameter. Photo- $z$ estimates in the present work have a mean error of $\sim 10 \%$ and show good agreement with the available spectroscopic measurements (Sect. 4.2). In order to estimate the impact of the photo- $z$ uncertainty on the measured physical parameters we offset the redshifts (Table 2) by their $1 \sigma$ errors to both sides and rerun the iterative physical parameter estimation procedures (see Sect. 3.3).

We find that, for the flux $f_{\mathrm{X}}\left(<r_{500}\right)$, all values are consistent within their $1 \sigma$ uncertainty and for most clusters the relative difference is below the $\sim 2 \%$ level (Fig. 15, left). Change in the photo- $z$ affects the flux in a complex way - it is entering directly the energy-conversion factor (ECF) calculation (lower redshift leads to a lower ECF), and also during the iterative process through the scaling relations, which then feed back into the aperture size itself as well as the temperature which again affects the ECF value. This complex dependence explains the scatter of the flux residuals in Fig. 15, leading to different convergence points for different input photo-zs. Interestingly, a lower photo- $z$ value leads to a higher flux in the $r_{500}$ aperture. The reason is that the direct effect of decreasing the photo- $z$ would be to lower temperature and mass and thus also reduce the $r_{500}$ value. However, the redshift dependence of the angular distance is stronger and thus the angular size of the $r_{500}$ aperture is actually larger for lower redshifts, which leads to the increase in the $f_{\mathrm{X}}\left(<r_{500}\right)$ values (we confirm this explanation by checking the flux in fixed sky apertures).

For luminosities the photo- $z$ errors translate into a $\sim 20 \%$ uncertainty (Fig. 15, right). Here the dependence is dominated by the cosmological redshift dimming and thus higher redshifts yield also higher luminosities. If we now use the perturbed redshift and luminosity values to recalculate temperatures and masses, we find that the $T_{500}$ values vary on the $\sim 5 \%$ level, while for $M_{500}$ the uncertainty is on the $\sim 7 \%$ level.

2. In the present work, we have utilized the bolometric luminosity scaling relations of Pratt et al. (2009) based on the REXCESS cluster sample (Böhringer et al. 2007). These scaling relations are best suited for our purposes for several reasons. They were derived from XMM-Newton observations (removing possible calibration issues between relations derived from different instruments) of a representative cluster sample (REXCESS, Böhringer et al. 2007). The sample covers a great range of the cluster luminosity function without a bias towards a morphological structure type (like e.g. presence of a cooling core or merging activity). By using the bolometric relations we can also utilize the results 
R. Šuhada et al.: The XMM-BCS galaxy cluster survey. I.
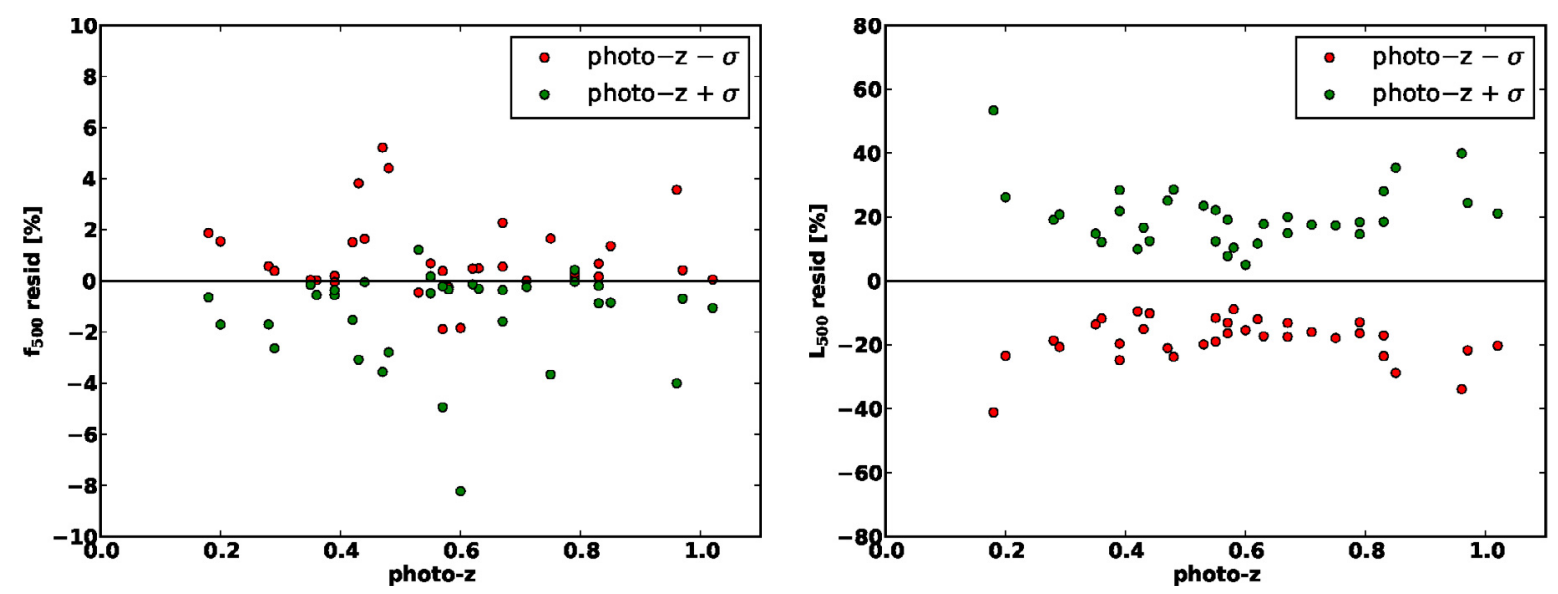

Fig. 15. Left: the effect of the photometric redshift uncertainty on the determined flux in the $r_{500}$ aperture. Right: the effect of the photometric redshift uncertainty on the determined luminosity in the $r_{500}$ aperture. Green points mark the relative difference of flux (luminosity) for photo- $z$ increased by $1 \sigma$ compared to the mean value. Red points are for the case when we decrease the photo- $z s$ by the same amount.

of Reichert et al. (2011) to estimate the effect of non-selfsimilar evolution on the estimated parameters (see below). Additionally, the $L-M$ relation is based on the $L-Y_{\mathrm{X}}$ and $Y_{\mathrm{X}}-M$ scaling relation, which is found to be more robust than previous direct $L-M$ calibrations (Arnaud et al. 2007). The direct application of these scaling relations, however requires extrapolations both to higher redshifts (the REXCESS cluster sample includes only local clusters with $z \lessgtr 0.2$ ) and to the low-mass regime of groups of galaxies. The physical parameters provided in Table 2 were obtained by assuming the redshift evolution of the cluster scaling relations to be self-similar. This is a standard assumption supported by predictions of a purely gravitationally driven cluster growth (e.g. Kaiser 1986). However, there is increasing evidence, that the evolution of the luminosity scaling relations is slower than the self-similar expectation (see Reichert et al. 2011, and references therein). We test the influence of this assumption by using the simplified approach proposed by Fassbender et al. (2011b) by removing a part of the selfsimilar evolution factor from the relations (the $L-T$ " no evolution" scenario). This approach is consistent with the more detailed analysis of Reichert et al. In this picture, the predicted temperatures are on average higher by $9 \%$ and masses by $15 \%$ compared to the self-similar scaling relations. At the high redshift end $(z>0.8)$ this effect is even more important ( $\sim 20 \%$ and $30 \%$ increase, respectively), while for $z<0.2$ the effect is less than $5 \%$.

The cluster sample of Vikhlinin et al. (2009) covers a similar redshift range as our sample (0-0.9) and extends in mass down to roughly the median mass of our sample $\left(\sim 1 \times 10^{14} M_{\odot}\right)$. Using the $L-M$ relation from this samples gives masses only slightly higher (by $\sim 5 \%$, standard deviation $\sim 13 \%$ ) than our results. This difference grows with redshift due to the deviation of the evolution index from the self-similar value (Vikhlinin et al. 2009 obtained $E(z)^{1.61}$ ).

Pertaining the assumptions that the REXCESS scaling relations hold also bellow $M_{500} \approx 1 \times 10^{14} M_{\odot}$, Sun et al. (2011) found very good agreement between the pressure profiles of their sample of groups and those derived from REXCESS. Leauthaud et al. (2010) used the groups detected in the COSMOS survey (Finoguenov et al. 2007) to calibrate the $L-M$ scaling relation with weak lensing mass measurements. Their evolution factor is consistent with the one found by Reichert et al. (2011), i.e. giving only slightly higher masses than the self-similar scenario. These findings give us an indication that the scaling relations employed in the present work are indeed adequate also in the low-mass clusters/groups regime.

3. The Galactic hydrogen column densities reported by the LAB HI survey (Kalberla et al. 2005) are systematically lower by $\sim 27 \%$ than the Dickey \& Lockman (1990) values in the whole survey area. The effect on the derived luminosities is, however, only marginal $(\sim 1.6 \%)$.

4. In order to quantify the effect of possible deviations of the cluster metallicity from the mean value of 0.3 solar, we bracket the possible metallicities in the very conservative range of $(0.1,0.6)$ solar. The corresponding range of $L_{X}\left(<r_{500}\right)$ deviations from the fiducial value (for 0.3 solar metallicity) is $(1.6 \%,-1.5 \%)$, i.e. lower metallicities lead to higher luminosities and vice versa.

5. We also test the quality of the flux extrapolation correction described in Sect. 3.3. The correction coefficients are calculated by integrating a beta model between $\left(r_{\text {plat }}, r_{500}\right)$, if $r_{\text {plat }}<r_{500}$. We use Eq. (1) to estimate the $\beta$ and $r_{\mathrm{C}}$ parameters. Alternatively, we can use the canonical value $\beta=2 / 3$ and $r_{\mathrm{C}}$ obtained from the maximum likelihood fit in the source detection step. The two extrapolation methods give fluxes (luminosities) differing on average for the whole sample by $2 \%$. For individual objects the relative difference of fluxes is clearly correlated with the amount of extrapolation needed and is roughly of the size of the correction itself. This means that the extrapolation is currently only very weakly constrained and thus highly uncertain. Fortunately, for most of our sample the required extrapolation factor is small.

6. The combined MOS1 and MOS2 counts are converted to flux and luminosities using the MOS2 response matrix (see Sect. 3.3). We have chosen the MOS2 response matrix over the MOS1, because some sources lie on the missing MOS1 CCD\#6, where no meaningful response matrix can be calculated. If the MOS1 response is used instead, the luminosities obtained purely from the combined MOS detectors are on average lower by $2 \%$ (excluding clusters detected on the position of the missing MOS1 chip). The final $L_{X}\left(<r_{500}\right)$ calculated as the weighted average of the individual PN and MOS luminosities is affected by less than $1.3 \%$.

7. The response matrices used in our analysis are calculated for a fixed radius of 150 arcsec. This range is roughly the average extraction radius of our clusters (i.e. from which 
growth curves are extracted and local background estimated). We calculate response matrices for two additional radii -60 arcsec and 240 arcsec, to check how the spatial averaging of the spectral response impacts the derived ECFs and thus flux and luminosity. In this very conservative range of extraction radii we found the average effect to be of the order of $2.5 \%$.

8. The uncertainties in the absolute normalization of the effective area of the detectors decrease the flux measurement precision. Nevalainen et al. (2011) found an agreement between $0.5-2 \mathrm{keV}$ fluxes measured by PN and both MOS cameras to be better than $\sim 5-7 \%$ and for ACIS on Chandra and the PN found the fluxes to differ only by $2 \%$.

9. We have tested the impact of using the up-to-date version of the SAS package (SAS v. 11.0.0), with respect to the SAS v. 7.1.0 adopted for the analysis in this paper. In an end-toend reprocessing of a subsample of six clusters fully sampling our flux and off-axis angle distributions we find on average only very small differences $(<4 \%$ in flux, $<1.5 \%$ in temperatures and $<2.5 \%$ in masses). These differences can get larger for low quality candidates (e.g. quality flag 3 in Table A.1), but in all test cases they were consistent within the error bars.

10. We also tried to run the physical parameter estimation procedure (Sect. 3.3) from several initial values of $T_{500} / r_{500}$. The iteration procedure always converged to the same solution, confirming its independence from the starting values.

We will provide tests of photometric accuracy of the growth curve method in a subsequent publication based on simulations using realistic backgrounds (i.e. using our survey fields as background for the simulated clusters). Presently we provide a comparison of the X-ray photometry obtained by our pipeline in comparison with the XMM-LSS project in Appendix C.1. In addition, our algorithm was also applied to the clusters SPT-CL J2332-5358 and SPT-CL J2342-5411 in Šuhada et al. (2010) detected in the extension of the XMM-BCS survey. These sources have been independently analysed by Andersson et al. (2011) using deeper pointed data (from a $19 \mathrm{ks}$ long XMM-Newton observation for the first cluster and from a $134 \mathrm{ks}$ long Chandra exposure for the second). Andersson et al. (2011) find for SPT-CL J2332-5358 $\left(z_{\text {photo }}=0.32\right)$ an X-ray luminosity $L_{500}=3.0 \pm 0.1 \times 10^{44} \mathrm{erg} \mathrm{s}^{-1}$ and for SPT-CL 2342-5411 $\left(z_{\text {photo }}=1.08\right) L_{500}=2.9 \pm 0.3 \times 10^{44} \mathrm{erg} \mathrm{s}^{-1}$ in good agreement with our values: $L_{500}=2.7 \pm 0.2 \times 10^{44} \mathrm{erg} \mathrm{s}^{-1}$ for SPT-CL 23325358 and $L_{500}=2.9 \pm 0.3 \times 10^{44} \mathrm{erg} \mathrm{s}^{-1}$ for SPT-CL 2342-5411 (luminosities in the $0.5-2 \mathrm{keV}$ band). Estimated temperatures and masses are also consistent within error bars although the uncertainties on these parameters are significant given the exposure time in the Šuhada et al. (2010) analysis was $\$ 3 \mathrm{ks}$.

A proper understanding of a realistic error budget of a cluster sample is crucial for its modelling in the cosmological context. From our analysis we find that most effects are typically on the $\sim 2 \%$ level (under conservative assumptions) and the major contributing factors are the uncertainty of the photo- $z$ measurements and the required extrapolations of the scaling relations (both in the range of 5-30\% depending on the parameter and the redshift of the system). For a few clusters an additional significant source of uncertainty is connected with the flux extrapolation. A full self-consistent treatment of the error propagation (including their full covariance matrices) and its impact on the cosmological modeling of the sample will be addressed in subsequent work.

\subsection{Project outlook}

The present sample establishes the observational base of the $\mathrm{X}$-ray part of the XMM-BCS survey. In upcoming work we will use the available multi-wavelength data to follow several lines of investigations, some of which have already been initiated:

- The X-ray cluster catalog will be extended to cover the whole $14 \mathrm{deg}^{2}$ area. The preliminary source catalog is already available and we will follow up this work by estimating the photometric redshifts and physical parameters for the clusters in the same way as presented in this work. The full cluster catalog is expected to comprise $\sim 100$ clusters and groups of galaxies.

- We will calculate the selection function based on Monte Carlo simulations developed by Mühlegger (2010). This analysis will allow us to construct a well controlled subsample from the full cluster catalog that will be suitable for cosmological modelling.

- A more detailed analysis of optical properties of the clusters presented in this sample will be provided in Song et al. (in prep.) We will provide here measurements of the $N_{200}$ and $L_{200}^{\mathrm{opt}}$ parameters and investigate their mass scaling relations.

- A detailed comparison of the X-ray, optical and mid-infrared cluster samples will allow us to gain good understanding of the selection function of each method. We will study the cluster/group population in this field and establish its multiwavelength properties. The Spitzer imaging data will also be used to improve the photometric redshift estimates, especially for distant systems with redshift $z \gtrsim 0.8$.

- We have initiated further X-ray-SZE studies based on a cooperation with the SPT collaboration. The current SPT cluster sample (Vanderlinde et al. 2010; Williamson et al. 2011) includes only sources with minimal detection significance of $5 \sigma(7 \sigma)$, respectively. There are only two clusters in the $14 \mathrm{deg}^{2}$ above this threshold, SPT-CL J2332-5358 and SPTCL J2342-5411, which are also independently detected in our survey (Šuhada et al. 2010). Using our X-ray selected cluster catalog we can also safely investigate lower significance SPT detections. As a first example, cluster ID 044, (XBCS 231653.1-545413) was found to have a direct SPT detection at the $4.2 \sigma$ level (Benson, priv. comm.). Another approach is a stacking analysis of the SZE data for the X-ray selected clusters. Here, a preliminary analysis of the top eleven clusters ranked by their X-ray predicted SPT detection significance yields a $\gtrsim 6 \sigma$ detection. We will explore both approaches in more depth in upcoming work, but already now it is clear, that with a joint SZE and X-ray analysis we are able to explore a completely new mass regime in the SPT survey.

- The multi-wavelength coverage of the field provides opportunities also for non-cluster science. As an example, we have detected a total of 3065 X-ray point sources in the survey (1639 in the core region and 1426 in the extension). Most of these point sources are AGN and using the available multiwavelength data we will be able to carry out a study with a focus on the obscured AGN population.

\section{Summary and conclusions}

- We have provided the analysis of the $6 \mathrm{deg}^{2} X M M-N e w t o n$ field in the framework of the XMM-BCS survey. We have carried out X-ray source detection and constructed a catalog of 46 clusters and groups of galaxies. 
- Based on four band optical imaging provided by the Blanco Cosmology Survey we have confirmed that these X-ray detections are coincident with overdensities of red galaxies. Using the red sequence method we have measured the photometric redshifts of these systems.

- We have initiated a spectroscopic follow-up program by carrying out long-slit spectroscopy observations using the EFOSC2 instrument at the $3.6 \mathrm{~m}$ NTT telescope at La Silla, Chile. We have obtained spectroscopic redshifts for BCG galaxies in 12 clusters and in four cases also for one additional member galaxy. This sample covers the redshift range $0<z<0.4$ (i.e. roughly up to the median redshift of the sample) and constitutes the first spectroscopic information for the field. We find good agreement between our photometric estimates and the spectroscopic values, but the spectroscopic sample has to be extended in redshift, in order to be able to provide a rigorous calibration of the photo- $z s$.

- Using the redshift information we measured the X-ray luminosities for our cluster sample. From luminosity scaling relations we estimate their most important physical parameters, e.g. mass, temperature and the $Y_{\mathrm{X}}$ parameter. We discuss the influence of several factors on the precision of the provided estimates. The uncertainty of the photometric redshift estimates and the extrapolation of the scaling relations to high redshift systems and into the group regime are identified as the most important factors that determine the overall errors in the physical parameters. We verify our X-ray parameter estimation method by analyzing the C1 sample of the XMMLSS survey (Pacaud et al. 2007). We find good agreement between the parameters provided by both pipelines.

- The present sample of clusters and groups of galaxies covers the redshift range from $z=0.1$ to redshift $z \approx 1$ with a median redshift of $z=0.47$. The median temperature of the clusters is $\sim 2 \mathrm{keV}$, and the median $M_{500}$ mass $9 \times 10^{13} M_{\odot}$ (based on luminosity scaling relations). With our $\sim 10 \mathrm{ks}$ $X M M$-Newton observations we are thus able to effectively probe the cluster/group transition regime practically at all redshifts up to $z \approx 1$.

- We provide a preliminary, simplified calculation of the survey sky coverage which does not require extensive Monte Carlo simulations. Using this calculation we characterize our cluster sample by its $\log N-\log S$ relation. We find good agreement with the relations established by the RDCS survey (Rosati et al. 1998), $400 \mathrm{deg}^{2}$ survey (Burenin et al. 2007; Vikhlinin et al. 2009) and the XMM-LSS project (Pacaud et al. 2007).

- We carried out first comparisons with optical studies available from the Southern Cosmology Survey (SCS, Menanteau et al. 2009, 2010). In this preliminary investigation we find the SCS photometric redshifts to be biased low by $\sim 20 \%$ with respect to our estimates (both photometric and spectroscopic, where available). We find a discrepancy between the X-ray and optical mass estimates, with optical masses being significantly higher. We compare our masses to weak lensing mass measurements available for 13 clusters in our sample from McInnes et al. (2009). Although the weak lensing mass uncertainties are large, there is no statistical inconsistency between the two mass estimators.

The presented results illustrate the potential of medium-deep, $\mathrm{X}$-ray surveys to deliver cluster samples for cosmological modelling. These samples then in combination with available multiwavelength data (particularly in optical, near-infrared and SZE) will allow us to probe the dependence of the selection functions on relevant cluster observables and provide thus an important input for upcoming large-area multi-wavelength cluster surveys.

Acknowledgements. We thank the referee for detailed comments on the manuscript. We are thankful to Bradford Benson for providing the preliminary SPT analysis. We thank Stefania Giodini and Veronica Biffi for carrying out GROND observations for several XMM-BCS clusters. We thank Rodion Burenin for providing the $400 \mathrm{deg}^{2}$ survey $\log N-\log S$ relation and Hermann Brunner for useful discussions. We are thankful to Martin Pančišin and Alexandra Weißmann for their comments on the manuscript. R.S. acknowledges support by the DfG in the program SPP1177. H.B. acknowledges support for the research group through The Cluster of Excellence "Origin and Structure of the Universe", funded by the Excellence Initiative of the Federal Government of Germany, EXC project number 153 . This research has been partially supported through a NASA grant NNX07AT95G to UMBC. D.P. acknowledges the kind hospitality of the Max-Planck-Institute for extraterrestrial Physik. This research has made use of the NASA/IPAC Extragalactic Database (NED) which is operated by the Jet Propulsion Laboratory, California Institute of Technology, under contract with the National Aeronautics and Space Administration.

\section{Appendix A: Ancillary information}

\section{A.1. Quality flags}

In this section we provide useful ancillary data for our clusters described using several X-ray quality flags and diagnostic parameters compiled in Table A.1. Here is the description of the table's columns:

- ID: the cluster identification number.

- BCS field: the identification number of the BCS field, on which the cluster is lying. Some clusters lie on two or more tiles, in those cases we provide the name of the tile with the largest overlap region.

- XMM OBSID: The official identification number of the $X M M-N e w t o n$ pointing containing the cluster. If the cluster lies in two (or three) adjacent observations we provide the OBSID of the pointing which provides the best constraint on the cluster flux (typically the one where the cluster is at the smallest off-axis angle).

- Internal field ID: XMM-BCS internal field ID used in the text as shorthand for the OBSID. See also Table 1 for the full cross-listing of field IDs.

- flag $_{\mathrm{HC}}$ : The hot chip flag is a four character string, with the characters being either T for "true" or F for "false". The significance of the characters:

1. character: Does the observation have a hot MOS2 CCD\#5?

2. character: Does the cluster lie on the MOS2 CCD\#5?

3. character: Does the observation have a hot MOS1 $\mathrm{CCD} \# 4$ ?

4. character: Does the cluster lie on the MOS1 CCD\#4?

For the problematics of the hot chips see Sect. 3.1.1.

- $S N R_{\mathrm{Xflux}}$ : The flux estimation significance determined as $F_{500} / \sigma_{F_{500}}$, where $F_{500}$ is the source flux in the $r_{500}$ aperture, and $\sigma_{F_{500}}$ is its error (including shot noise and 5\% background modelling uncertainty, Sect. 3.2).

- flag $_{\text {inst }}$ : The instrument flag equals 0 if the physical parameters of the source were obtained using the PN and both MOS cameras. If flag inst $=1$ only PN could be used and if flag $_{\text {inst }}=2$ only the combination of the two MOS cameras was utilized.

- $Q_{\text {plat }}^{\text {PN }}$ The automatic plateau fit quality flag for the PN growth curves. These flags have the same meaning as in Böhringer et al. (2000). In summary, as described in Sect. 3.2 we fit a line to the growth curve between $r_{\text {plat }}$ and the outer extraction radius. The flag describes the quality of this fit by calculating the ratio of the predicted count rate from the linear fit 
R. Šuhada et al.: The XMM-BCS galaxy cluster survey. I.

Table A.2. Galaxies identified in the NED database to be within $16^{\prime \prime}$ from the X-ray center.

\begin{tabular}{clcccc}
\hline \hline ID & \multicolumn{1}{c}{ Object name } & RA (deg) & Dec $(\mathrm{deg})$ & Redshift & Separation \\
\hline 034 & APMUKS(BJ) B232750.10-560012.1 & 352.6544 & -55.7274 & & $1.9^{\prime \prime}$ \\
039 & 2MASX J23191712-5519284 & 349.8214 & -55.3245 & & $0.5^{\prime \prime}$ \\
041 & 2MASX J23190212-5523195 & 349.7588 & -55.3888 & & $1.3^{\prime \prime}$ \\
070 & 2MASX J23223092-5416086 & 350.6289 & -54.2691 & & $0.8^{\prime \prime}$ \\
094 & APMUKS(BJ) B232918.62-552918.2 & 353.0196 & -55.2122 & & $2.5^{\prime \prime}$ \\
127 & 2MASX J23272468-5503589 & 351.8528 & -55.0664 & & $9.6^{\prime \prime}$ \\
150 & 2MASX J23300047-5437069 & 352.5019 & -54.6187 & $0.177^{a}$ & $1.5^{\prime \prime}$ \\
152 & 2MASX J23294006-5447220 & 352.4168 & -54.7895 & & $3.1^{\prime \prime}$ \\
227 & APMUKS(BJ) B231920.30-554137.6 & 350.5444 & -55.4194 & & $4.4^{\prime \prime}$ \\
268 & APMUKS(BJ) B232326.14-554657.3 & 351.5618 & -55.5074 & & $14.0^{\prime \prime}$ \\
476 & 2MASX J23254015-5444308 & 351.4173 & -54.7419 & $0.101^{a}$ & $3.1^{\prime \prime}$ \\
511 & APMUKS(BJ) B232929.68-545847.0 & 353.0645 & -54.7035 & & $10.8^{\prime \prime}$ \\
547 & 2MASX J23241957-5525494 & 351.0816 & -55.4303 & & $0.6^{\prime \prime}$ \\
\hline
\end{tabular}

Notes. We list spectroscopic redshifts where available - in both cases the identified galaxies are BCGs. Redshift reference: ${ }^{(a)}$ Jones et al. (2004).

to the expectation, if the plateau was constant and equal to the estimated plateau flux. $Q_{\text {plat }}^{\mathrm{PN}}=1$ : the growth curve shows neither significant increase nor decrease outside $r_{\text {plat }}$. This value is assigned if the linear extrapolation does not differ by more than $0.8 \%$ per bin from the constant value. $Q_{\text {plat }}^{\mathrm{PN}}=2$ : marks a declining curve (decline $>0.8 \% /$ bin). A decline can occur if the background model (determined from a fit to the whole field) slightly overestimates the local background. In this case we attempt to estimate the plateau level from the 3 bins closest to $r_{\text {plat }}$. If the final fit is acceptable (no significant residual decline), the plateau is accepted and assigned this quality flag. $Q_{\mathrm{plat}}^{\mathrm{PN}}=3$ and $Q_{\mathrm{plat}}^{\mathrm{PN}}=4$ : in case that the plateau is rising an attempt is made to iteratively exclude the outermost bins and in a second step also the innermost bins. This procedure helps in correcting an outer rise of the growth curve due to a neighboring source and if necessary by skipping over a few bins if the curve fluctuates in the radial range close to $r_{\text {plat }}$. If this procedure converges, after the exclusion of the outermost bins the plateau is accepted and flagged with $Q_{\mathrm{plat}}^{\mathrm{PN}}=3$. If the procedure converges, but it required also the second step of excluding the innermost bins we assign $Q_{\mathrm{plat}}^{\mathrm{PN}}=4$. If $Q_{\text {plat }}^{\mathrm{PN}}=5$, the plateau is rising and the increase could not be corrected for by the above described procedure. If there are only two or less radial bins outside the plateau radius can not be established and we assign $Q_{\mathrm{plat}}^{\mathrm{PN}}=9$.

$Q_{\mathrm{plat}}^{\mathrm{PN}} \leq 4$ mark generally good quality plateaus (naturally, the lower the flag the better). $Q_{\mathrm{plat}}^{\mathrm{PN}}=5$ is a serious warning and $Q_{\text {plat }}^{\mathrm{PN}}=9$ is not recommended to be used at all. In fact, for the parameters in Table 2 we do not use plateaus with this flag with the exception for the systems ID 476 and 139 where an alternative solution is not available due to their significant blending (Appendix A.2).

- $Q_{\text {plat }}^{\text {MOS}}$ : The same as $Q_{\text {plat }}^{\mathrm{PN}}$ but applied to the MOS1+MOS2 growth curve.

- $Q_{\mathrm{gca}}^{\mathrm{PN}}$ : Visual flag set considering the overall quality of the PN growth curve solution (taking into account the presence of chip gaps, anomalous background, potential contamination etc.). Value equal to 1 is the best (no problems), equal to 3 the worse (to be considered as a warning). If the given detector was not included in the analysis (as marked by flag ${ }_{\text {inst }}$ ) $Q_{\mathrm{gca}}^{\mathrm{PN}}$ is set to 9 .
- $Q_{\mathrm{gca}}^{\mathrm{MOS}}$ : The same as $Q_{\mathrm{gca}}^{\mathrm{PN}}$ but applied to the MOS1+MOS2 growth curve.

- $Q_{\text {Tот: }}$ This flag is a non-quantitative ancillary X-ray flag. It is assigned during a visual inspection of each source, but takes into consideration also all above flags. Sources with this flag equal to 1 (best) and 2 (only mild warnings) have high quality X-ray photometry measurements. Flag equal to 3 signifies problems with the X-ray photometry e.g. due to problematic locations on the detectors, bad quality plateaus, source blending etc. In the present work, we have included also $Q_{\text {TOT }}=3$ sources in all analyses since they constitute only about $\sim 10 \%$ of the sample.

\section{A.2. Notes on individual sources}

Some of the identified clusters required individual treatment and in this section we provide notes for these cases:

- ID 011: this high redshift cluster lies on the heavily flared field F03 with no quiescent period. Therefore, the field was not used for the sensitivity function calculation and the $\log N-\log S$. The double component background model accounts in principle in the first approximation for the enhanced background and therefore we provide the basic X-ray parameters for this cluster. The diagnostic flags (Table A.1) indicate that the growth curve solution is quite reliable, but due to the flaring all physical parameters should be treated with caution.

- ID 038: this source consists of two completely overlapping systems, one with photometric redshift $z=0.39 \pm 0.05$ and the second with $z=0.74 \pm 0.07$. Since there is no direct way to disentangle the contribution of the two sources, we will assume that all the flux comes from the more nearby system. In this case, the estimated physical parameters are upper limits.

- ID 070: is a nearby cluster with large extent and measured flux. It lies on a hot MOS2 CCD\#5 and due to its extent it is impossible to obtain a background area on this chip uncontaminated by the source emission. Therefore we can not use the procedure described in Sect. 3.1.1, where we fit a double component model to the hot chip independently from the rest of the field. Instead we discard the data from this chip 


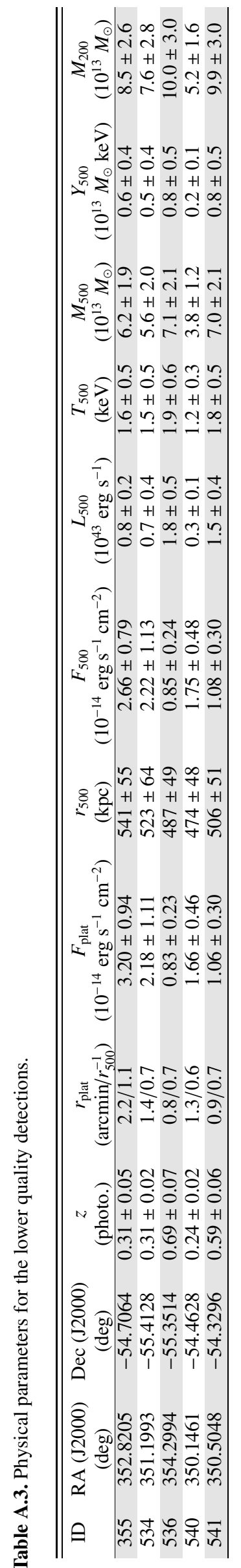

completely. Consistently, we use the MOS1 response matrix instead of the MOS2 one for further analysis.

- ID 081: in this distant cluster (photo- $z=0.85$ ) the BCG is offset by $\sim 19^{\prime \prime}$ from the X-ray centroid. The emission from this system might be contaminated by an intervening AGN (the X-ray center is coincident with a bright galaxy which could harbor one).

- ID 109: due to the limited depth of the available optical data we can provide only very tentative redshift estimate for this system.

- ID 139 and 476: we detect two nearby, high significance extended sources in this region $(\sim 1.3$ arcmin apart). The systems are confirmed as independent also in redshift space by our spectroscopic measurements (ID 476 at $z=0.102$ and ID 139 at $z=0.169)$. In order to measure the flux of each cluster we excise the other source. Due to their proximity, however, full deblending is not possible and therefore both fluxes are likely overestimated. The analysis of the sources is further complicated by the presence of a very bright X-ray point source at $\sim 2$ arcmin distance from the clusters and high quiescent soft proton contamination in the PN camera (39\% background increase, Sect. 2).

The cluster catalog of Burenin et al. (2007) based on ROSAT data includes a source with a center roughly between the two systems (i.e. very likely misclassified as a single cluster due to the limited resolution of ROSAT).

- ID 275: also lies on a hot MOS2 CCD\#5. Its detection likelihood is completely dominated by the MOS2 detection, however the source is not flagged as spurious based on the criteria described in Sect. 3.1.1, because it would be above the detection threshold even without the MOS2 data. In this case, the background modeling of the hot chip was possible and this background was used in the subsequent growth curve analysis.

\section{A.3. Galactic counterparts}

All NED galactic counterparts within 16" from the X-ray centroids of our clusters are summarized in Table A.2 (see also Sect. 5.4).

\section{A.4. Wavelet detections}

We have also carried out a detection using a wavelet detection algorithm developed for the COSMOS project by Finoguenov et al. (2007, 2009). Every cluster presented in our current sample has also been confirmed by this approach. In addition, we have identified five systems (Table A.3) with a wavelet detection but no SAS-based detection in any setup. All five systems are coincident with significant galaxy overdensities. We find many interloping X-ray point-sources in these systems (a potential source of misclassification in the SAS detections). Even after conservative point source removal, residual contamination makes all the estimated X-ray parameters for these systems highly uncertain. These detections are not included in the statistical description of the sample (e.g. the $\log N-\log S$ relation) and are listed here only for completeness (in Table A.3).

\section{Appendix B: Test of the sensitivity function from preliminary Monte Carlo simulations}

The determination of the survey selection function is a crucial requirement for the cosmological modeling of the cluster sample, 
scaling relation studies etc. Due to the complex nature of the extended source detection, this question can be properly addressed only by detailed Monte Carlo simulations. In the present work we utilized a simplifying approach that allowed us to get a first estimate of the sensitivity functions and the recovered $\log N-\log S$ relation (Sects. 5.2 and 5.3).

The software for the Monte Carlo simulations (Mühlegger 2010) is in an advanced development stage which allows us to carry out a preliminary test of our simplified approach.

The simulation pipeline uses the survey fields themselves and injects mock beta model clusters into the observations at random positions across the field-of-view. The field is then processed with the detection pipeline. The process is repeated on a grid of cluster fluxes and core radii and the cluster detection probability is derived as a function of these parameters. The use of real observations instead of model backgrounds allows us to derive a realistic selection function. The simulation software is described in detail in Mühlegger (2010).

Simulations are currently available for a subset of the XMM-Newton Distant Cluster Project (XDCP, Böhringer et al. 2005; Fassbender 2008; Fassbender et al. 2011a) fields. From these fields we selected 3 observations (XMM OBSIDs 0104860201, 0111970101, 0112551101) which have similar depth to our survey fields (e.g. cleaned exposure times $\sim 10 \mathrm{ks}$ and enough area unaffected by the central source to safely assess the background). We processed these fields with our detection pipeline and calculated the point source sensitivity function and the scaled extended sensitivity function as described in Sect. 5.2. The comparison with the sensitivity function derived from the simulations are displayed in Fig. B.1. The simple calculation (black curves) already matches the realistic calculation (red curves) very well, capturing also the transition parts of the curve. The curves from simulations include the effect of incompleteness of the output catalogs. The red curves in Fig. B.1 are calculated for a $50 \%$ completeness level $(c=0.5)$. The completeness of our cluster catalog can be assessed only by simulations, but is certainly higher than $50 \%$. This means that the preliminary analytic sky coverage function overestimates the sky coverage. The use of the true sky-coverage function would lead to an increase of the weighting factor in Eq. (5) and would move the points in Fig. 12 in the relevant flux range slightly higher. The sensitivity function for a $90 \%$ completeness scenario is plotted in green and as expected yields a much smaller area for the given flux.

Additional subtle effects slightly influence this comparison, e.g. leading to different normalizations of the two curves in the saturated high-end part: 1) All the fields have a bright source in the center of the field-of-view, which has to be excised. The excision is treated slightly differently in the simulations and in the simple calculations leading to slightly different total geometric areas. 2) The simulated curves were calculated for the single band detection scheme in the $0.35-2.4 \mathrm{keV}$ while our analytic solution is for the $0.5-2 \mathrm{keV}$ band. The fluxes were converted to the $0.5-2 \mathrm{keV}$ band, but detection in these different energy ranges could cause slightly different completeness and contamination fractions.

We conclude, that our first-order approach yields a good description of the sensitivity function for a $50 \%$ completeness level. To estimate the completeness of our sample Monte Carlo simulations are needed. The sensitivity functions from Sect. 5.2 provides sufficient precision for present applications and the preliminary $\log N-\log S$ is already in good agreement with previous findings. The described simulation pipeline will be applied to the whole XMM-BCS survey in subsequent work and the
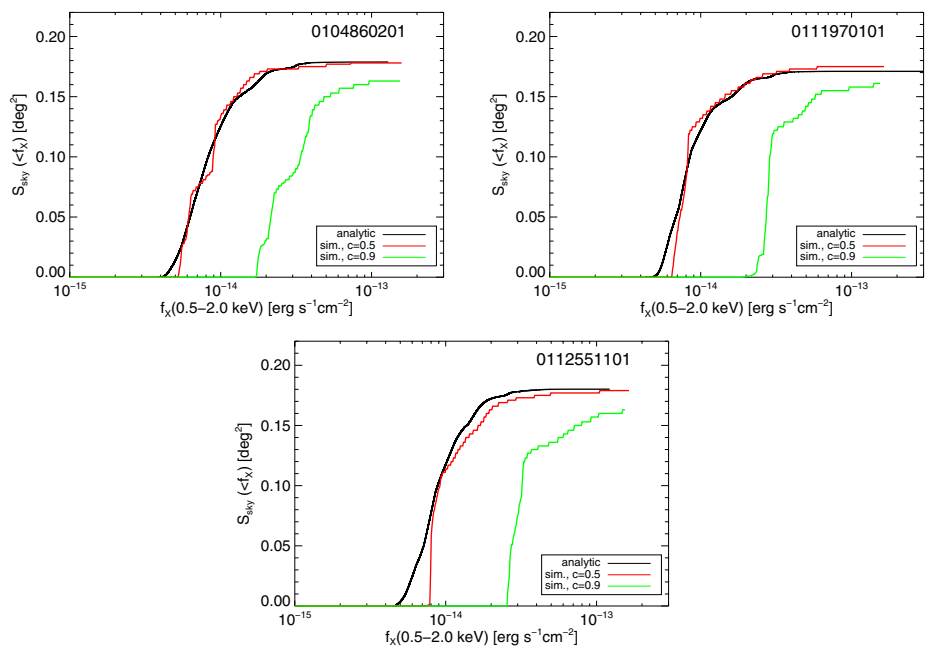

Fig. B.1. Sky-coverage for extended sources in three XDCP fields from Monte Carlo simulations at the 50\% (red curve) and 90\% (green) completeness level. The black solid curve shows the sky-coverage calculated by scaling the point source curve (dashed) with an offset factor of 2.4 (see Sect. 5.2). The simple scaling is shown to be a good first order description of the extended source sensitivity function. See Appendix B and Sect. 5.2 for details.

realistic selection function will be utilized for further analysis and modelling of the final cluster sample.

\section{Appendix C: Comparison with the XMM-LSS survey}

The first part of the XMM-LSS survey (the initial $5 \mathrm{deg}^{2}$, Pierre et al. 2007; Pacaud et al. 2006, 2007) offers an excellent match to our survey not only with respect to the area, but also to the typical depth (having only slightly higher average exposure times). Since the XMM-LSS project has already carried out Monte Carlo simulations to calibrate its detection and sourcecharacterization pipeline, we make here an effort to compare results derived from our XMM-BCS pipeline with results published by XMM-LSS.

\section{C.1. Cluster detection comparison}

A full comparison of the source detection pipelines would be only of limited use and is currently impossible since only a small part of the XMM-LSS extended sources have been thus far spectroscopically confirmed (the so-called $\mathrm{C} 1$ sample of Pacaud et al. $2007)^{14}$. Therefore, we restrict ourselves to the reanalysis of the C1 sample.

We downloaded all the XMM-LSS fields with C1 detections ${ }^{15}$ and fully reanalyzed them with the XMM-BCS pipeline. We confidently detected all the $\mathrm{C} 1$ clusters and they are among our highest ranked extended source detections.

In Fig. C.1 we compare their detection and extent likelihoods with their respective XMM-LSS variants (SB_Detect_Likelihood and SB_Extent_Likelihood). Both sets of parameters exhibit a strong correlation, showing a

14 Catalog available at: heasarc.gsfc.nasa.gov/W3Browse/all/ xmmlssoid.html

15 XMM OBSIDs: 0037980301, 0037980701, 0037981001, 0037981101, 0037981201, 0037981501, 0037981601, 0037981801, 0037982501, 0037982601, 0109520201, 0109520301, 0109520601, 0111110301, 0111110401, 0112680101, 0112680201, 0112680301, 0112680401, 0112680501, 0147110101, 0147110201. 



Fig. C.1. Comparison of detection (left panel) and extent likelihoods (right panel) between our pipeline ( $x$-axis) and the XMM-LSS pipeline ( $y$-axis, Pacaud et al. 2007). The derived likelihoods are well correlated and the red line shows the best fit relations.

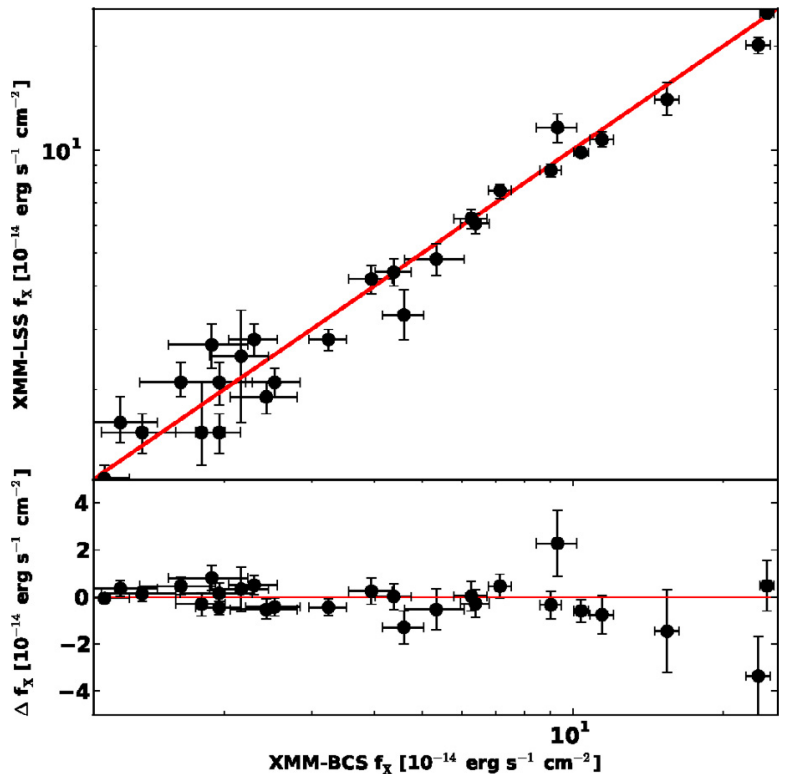

Fig. C.2. Comparison of measured $\mathrm{X}$-ray fluxes for the $\mathrm{C} 1$ subsample of the XMM-LSS survey in the $0.5-2 \mathrm{keV}$ band and a $0.5 \mathrm{Mpc}$ aperture (Pacaud et al. 2007, $y$-axis) and the fluxes measured by our pipeline ( $x$ axis). The red line marks equality. The bottom panel shows the residuals $\Delta f_{\mathrm{X}}=f_{\mathrm{X}}^{X M M-L S S}-f_{\mathrm{X}}^{X M M-B C S}$. See Sect. C. 2 for details.

good consistency between both detection approaches (XMMLSS uses a single band wavelet detection scheme). The scatter between the parameters is caused by differences in the data reduction process, background estimation and source detection algorithms.

The C1 sample is defined by SB_Detect_Likelihood $>32$, SB_Extent_Likelihood> 33. We fit a linear relation in the two log-log planes and use these cuts to convert the XMM-LSS thresholds to our parameters obtaining: det_ml> 16.4 (equivalent to $\sim 5.4 \sigma$ detection in our scheme) and ext_ml $>8.3$ (i.e. $\sim 3.7 \sigma$ extent significance).

\section{C.2. X-ray photometry comparison}

In Fig. C. 2 we compare the fluxes in the $0.5-2 \mathrm{keV}$ band and $0.5 \mathrm{Mpc}$ aperture measured by the XMM-LSS and by us using the growth curve method (Sect. 3.2). Being interested in the flux estimation we have fixed the cluster redshifts to its spectroscopic

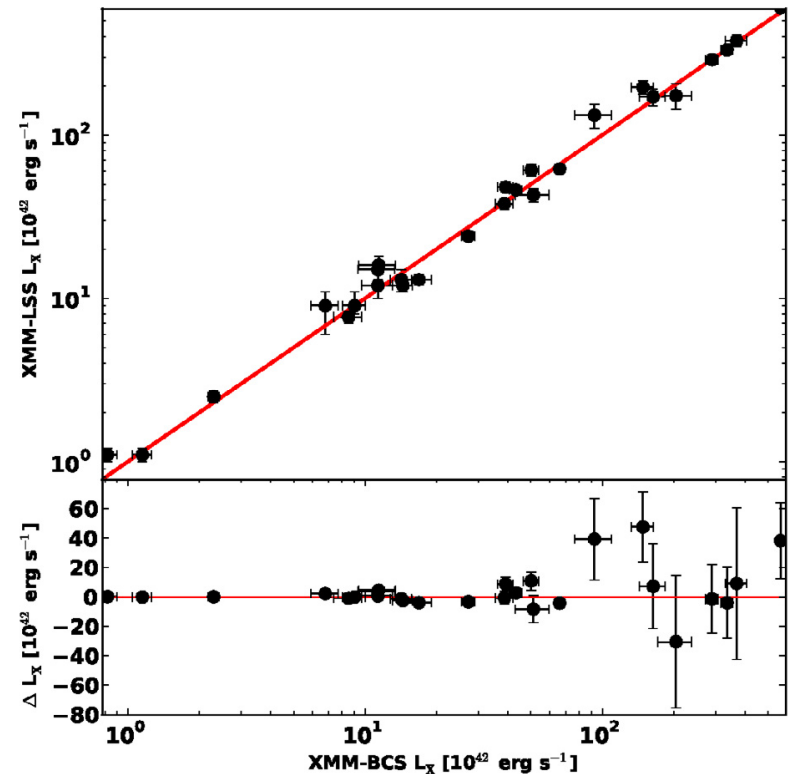

Fig. C.3. Comparison of bolometric X-ray luminosities for the $\mathrm{C} 1 \mathrm{sub}$ sample of the XMM-LSS survey in the $r_{500}$ aperture (Pacaud et al. 2007, $y$-axis) and the luminosities measured by our pipeline ( $x$-axis). The red line marks equality. The bottom panel shows the residuals $\Delta L_{\mathrm{X}}=L_{\mathrm{X}}^{\mathrm{XMM}-\mathrm{LSS}}-L_{\mathrm{X}}^{\mathrm{XMM}-\mathrm{BCS}}$. See Sect. C. 2 for details.

value provided by XMM-LSS. We choose not to use the information on the spectroscopic temperature, but rather we estimate it from the scaling relation as we do for the XMM-BCS sample (see Sect. 3.3).

The $0.5-2 \mathrm{keV}$ fluxes in a $0.5 \mathrm{Mpc}$ aperture are compared in Fig. C.2, where the residuals in the bottom panel are defined as $\Delta f_{\mathrm{X}}=f_{\mathrm{X}}^{\mathrm{XMM}-\mathrm{LSS}}-f_{\mathrm{X}}^{\mathrm{XMM}-\mathrm{BCS}}$. We find the fluxes in agreement with the mean relative difference of $\sim 0.6 \%$ and a scatter of $\sim 17 \%$ (averaged over the whole sample). If we split the sample by the median flux $\left(f_{\mathrm{x}} \approx 3.9 \times 10^{-14} \mathrm{erg} \mathrm{cm}^{-2} \mathrm{~s}^{-1}\right)$ the XMMLSS fluxes are on average by $\sim 4 \%$ higher on the faint end while being slightly lower by roughly the same amount on the brighter end. A systematic difference of this magnitude would however cause only a $\sim 1 \%$ shift in the temperature estimates and $\sim 2 \%$ shift on mass. An analogous comparison for the bolometric luminosity in the $r_{500}$ aperture is displayed in Fig. C.3. Here the mean relative difference is $\sim 6.6 \%$ and a scatter of $\sim 19 \%$ (the corresponding temperature/mass difference are $2 \% / 3 \%$ ). 


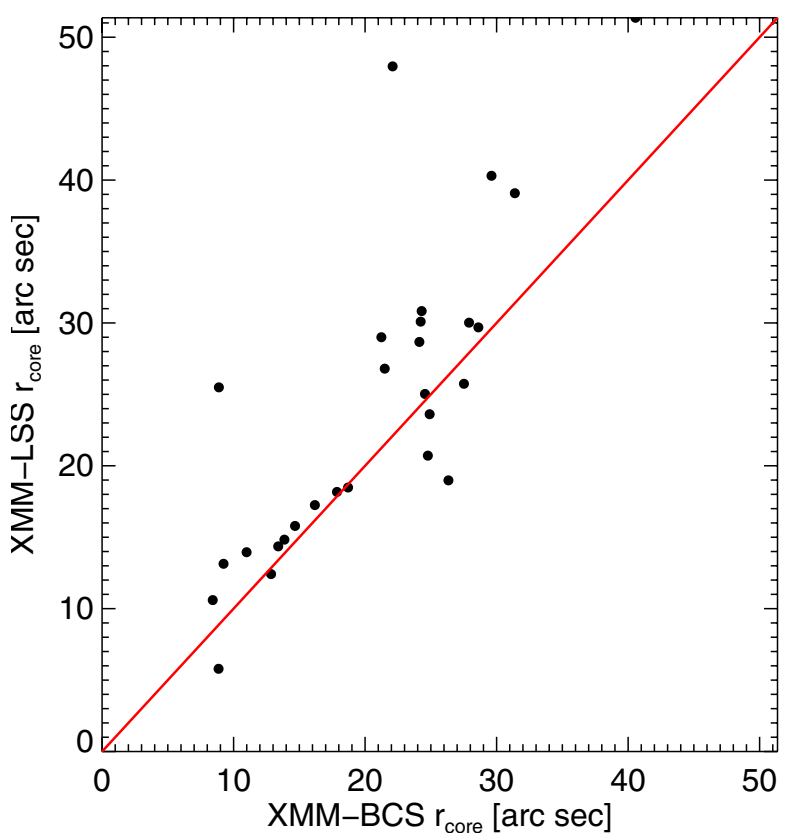

Fig. C.4. Beta model core radii for the XMM-LSS C1 sample as estimated by our pipeline ( $x$-axis) and by the XMM-LSS. The red line marks equality. The core radii are typically highly uncertain given the relatively low photon statistics. Despite this, the agreement between the two estimates is good. Note that the XMM-LSS values are fitted with the beta value as a free parameter, while we fix its value to $2 / 3$.

The overall agreement is encouraging, if we take into account that the two pipelines utilize principally different approaches to the flux measurement. XMM-LSS utilizes a beta model fit to the cluster's surface brightness integrated out to a fiducial radius, while our method is completely non-parametric (except for a typically small extrapolation factor if the required aperture is larger than the range where the cluster emission is detected directly). Background estimation in both approaches is also markedly different.

Since we did not use the information on the spectroscopic $\mathrm{X}$-ray temperature, it is interesting to note, that the mean temperature residuals (spectroscopic compared to our estimates from the scaling relations) are $<2 \%$ with a standard deviation of $\sim 23 \%$ (i.e. comparable to measurement errors). Although the error bars are large, this agreement indicates that the $L-T$ scaling relation and its evolution adopted in this work (Pratt et al. 2009) are suitable for cluster samples drawn from surveys of this type.

The cluster mass is not a direct observable in either of the two surveys. XMM-LSS gives rough estimates based on their spectroscopic measurement and beta model fit using the relation from Ettori (2000). Our estimates, using the $L-M$ relation of Pratt et al. (2009), give on average almost 40\% higher masses. We note however, that the Ettori (2000) relation was derived before the advent of XMM-Newton and Chandra. It is thus not well representing our current understanding of cluster mass estimation.

Finally, we also check the consistency of the beta model fits between the two pipelines. Since the core radius $r_{\text {core }}$ and the $\beta$ exponent of the beta model are strongly degenerate, especially for the case of low counts profiles, our fitting procedure keeps $\beta$ fixed to the canonical value of $2 / 3$. The XMM-LSS pipeline carries out fits with both $r_{\text {core }}$ and $\beta$ as free parameters. Despite this difference, we find good agreement between the estimated core radii (Fig. C.4).

\section{References}

Akritas, M. G., \& Bershady, M. A. 1996, ApJ, 470, 706

Andersson, K., Benson, B. A., Ade, P. A. R., et al. 2011, ApJ, 738, 48

Armstrong, B., Mohr, J., Adams, D., et al. 2010, in BAAS, 42, AAS Meet. Abstr. 215, 438.07

Arnaud, M., Pointecouteau, E., \& Pratt, G. W. 2007, A\&A, 474, L37

Arnaud, M., Pratt, G. W., Piffaretti, R., et al. 2010, A\&A, 517, A92

Benítez, N. 2000, ApJ, 536, 571

Bertin, E. 2006, in Astronomical Data Analysis Software and Systems XV, ed. C. Gabriel, C. Arviset, D. Ponz, \& S. Enrique, ASP Conf. Ser., 351, 112 Bertin, E., \& Arnouts, S. 1996, A\&AS, 117, 393

Bielby, R. M., Finoguenov, A., Tanaka, M., et al. 2010, A\&A, 523, A66

Böhringer, H., Voges, W., Huchra, J. P., et al. 2000, ApJS, 129, 435

Böhringer, H., Mullis, C., Rosati, P., et al. 2005, The Messenger, 120, 33

Böhringer, H., Schuecker, P., Pratt, G. W., et al. 2007, A\&A, 469, 363

Böhringer, H., Pratt, G. W., Arnaud, M., et al. 2010, A\&A, 514, A32

Bonamente, M., Joy, M., LaRoque, S. J., et al. 2008, ApJ, 675, 106

Bower, R. G., Lucey, J. R., \& Ellis, R. S. 1992, MNRAS, 254, 601

Bruzual, G., \& Charlot, S. 2003, MNRAS, 344, 1000

Bullock, J. S., Kolatt, T. S., Sigad, Y., et al. 2001, MNRAS, 321, 559

Burenin, R. A., Vikhlinin, A., Hornstrup, A., et al. 2007, ApJS, 172, 561

Cash, W. 1979, ApJ, 228, 939

Cavaliere, A., \& Fusco-Femiano, R. 1976, A\&A, 49, 137

da Silva, A. C., Kay, S. T., Liddle, A. R., \& Thomas, P. A. 2004, MNRAS, 348, 1401

De Luca, A., \& Molendi, S. 2004, A\&A, 419, 837

Dickey, J. M., \& Lockman, F. J. 1990, ARA\&A, 28, 215

Ettori, S. 2000, MNRAS, 311, 313

Fassbender, R. 2008, Ph.D. Thesis, Ludwig-Maximilians-Universitaet Muenchen [arXiv:0806.0861]

Fassbender, R., Böhringer, H., Nastasi, A., et al. 2011a, New J. Phys., submitted Fassbender, R., Böhringer, H., Santos, J. S., et al. 2011b, A\&A, 527, A78 Finoguenov, A., Guzzo, L., Hasinger, G., et al. 2007, ApJS, 172, 182

Finoguenov, A., Connelly, J. L., Parker, L. C., et al. 2009, ApJ, 704, 564

Gregory, P. C., Vavasour, J. D., Scott, W. K., \& Condon, J. J. 1994, ApJS, 90, 173

Haiman, Z., Mohr, J. J., \& Holder, G. P. 2001, ApJ, 553, 545

Haiman, Z., Allen, S., Bahcall, N., et al. 2005 [arXiv:0507013]

High, F. W., Stalder, B., Song, J., et al. 2010, ApJ, 723, 1736

Hincks, A. D., Acquaviva, V., Ade, P. A. R., et al. 2010, ApJS, 191, 423

Hu, W., \& Kravtsov, A. V. 2003, ApJ, 584, 702

Jeltema, T. E., Hallman, E. J., Burns, J. O., \& Motl, P. M. 2008, ApJ, 681, 167

Johnston, D. E., Sheldon, E. S., Wechsler, R. H., et al. 2007 [arXiv: 0709. 1159]

Jones, D. H., Saunders, W., Colless, M., et al. 2004, MNRAS, 355, 747

Kaastra, J. S. 1992, Internal SRON-Leiden Report, Updated Version 2.0, 1

Kaiser, N. 1986, MNRAS, 222, 323

Kalberla, P. M. W., Burton, W. B., Hartmann, D., et al. 2005, A\&A, 440, 775

Kravtsov, A. V., Vikhlinin, A., \& Nagai, D. 2006, ApJ, 650, 128

Kuntz, K. D., \& Snowden, S. L. 2008, A\&A, 478, 575

Leauthaud, A., Finoguenov, A., Kneib, J., et al. 2010, ApJ, 709, 97

Liedahl, D. A., Osterheld, A. L., \& Goldstein, W. H. 1995, ApJ, 438, L115

Maddox, S. J., Efstathiou, G., Sutherland, W. J., \& Loveday, J. 1990, MNRAS, 243, 692

Majumdar, S., \& Mohr, J. J. 2003, ApJ, 585, 603

Mantz, A., Allen, S. W., Ebeling, H., Rapetti, D., \& Drlica-Wagner, A. 2010a, MNRAS, 406, 1773

Mantz, A., Allen, S. W., Rapetti, D., \& Ebeling, H. 2010b, MNRAS, 406, 1759

Markevitch, M. 1998, ApJ, 504, 27

Marriage, T. A., Acquaviva, V., Ade, P. A. R., et al. 2011, ApJ, 737, 61

Marrone, D. P., Smith, G. P., Richard, J., et al. 2009, ApJ, 701, L114

Mauch, T., Murphy, T., Buttery, H. J., et al. 2003, MNRAS, 342, 1117

McInnes, R. N., Menanteau, F., Heavens, A. F., et al. 2009, MNRAS, 399, L84

Melin, J., Bartlett, J. G., Delabrouille, J., et al. 2011, A\&A, 525, A139

Menanteau, F., Hughes, J. P., Jimenez, R., et al. 2009, ApJ, 698, 1221

Menanteau, F., Hughes, J. P., Barrientos, L. F., et al. 2010, ApJS, 191, 340

Mewe, R., Gronenschild, E. H. B. M., \& van den Oord, G. H. J. 1985, A\&AS, 62, 197

Mohr, J. J., Adams, D., Barkhouse, W., et al. 2008, in SPIE Conf. Ser., 7016

Motl, P. M., Hallman, E. J., Burns, J. O., \& Norman, M. L. 2005, ApJ, 623, L63

Mühlegger, M. 2010, Ph.D. Thesis, Technische Universität München

Mullis, C. R., McNamara, B. R., Quintana, H., et al. 2003, ApJ, 594, 154

Nagai, D. 2006, ApJ, 650, 538

Navarro, J. F., Frenk, C. S., \& White, S. D. M. 1997, ApJ, 490, 493

Nevalainen, J., David, L., \& Guainazzi, M. 2011, A\&A, 523, A22

O’Hara, T. B., Mohr, J. J., Bialek, J. J., \& Evrard, A. E. 2006, ApJ, 639, 64

Pacaud, F., Pierre, M., Refregier, A., et al. 2006, MNRAS, 372, 578 
A\&A 537, A39 (2012)

Pacaud, F., Pierre, M., Adami, C., et al. 2007, MNRAS, 382, 1289

Pierre, M., Chiappetti, L., Pacaud, F., et al. 2007, MNRAS, 382, 279

Plagge, T., Benson, B. A., Ade, P. A. R., et al. 2010, ApJ, 716, 1118

Planck Collaboration 2011a, A\&A, 536, A8

Planck Collaboration 2011b, A\&A, 536, A10

Planck Collaboration 2011c, A\&A, 536, A11

Pratt, G. W., \& Arnaud, M. 2003, A\&A, 408, 1

Pratt, G. W., Croston, J. H., Arnaud, M., \& Böhringer, H. 2009, A\&A, 498, 361

Reichert, A., Böhringer, H., Fassbender, R., \& Mühlegger, M. 2011, A\&A, 535, A4

Reiprich, T. H. 2001, Ph.D. Thesis, Max-Planck-Institut für extraterrestrische Physik, PO Box 1312, 85741 Garching, Germany

Reiprich, T. H., \& Böhringer, H. 2002, ApJ, 567, 716

Reyes, R., Mandelbaum, R., Hirata, C., Bahcall, N., \& Seljak, U. 2008, MNRAS, 390, 1157

Rosati, P., della Ceca, R., Norman, C., \& Giacconi, R. 1998, ApJ, 492, L21
Scharf, C. 2002, ApJ, 572, 157

Skrutskie, M. F., Schneider, S. E., Stiening, R., et al. 2000, VizieR Online Data Catalog, 1, 2003

Skrutskie, M. F., Cutri, R. M., Stiening, R., et al. 2006, AJ, 131, 1163

Song, J., Mohr, J. J., Barkhouse, W. A., Warren, M. S., \& Rude, C. 2011, ApJ, submitted [arXiv: 1104.2332]

Staniszewski, Z., Ade, P. A. R., Aird, K. A., et al. 2009, ApJ, 701, 32

Sun, M., Sehgal, N., Voit, G. M., et al. 2011, ApJ, 727, L49

Sunyaev, R. A., \& Zel'dovich, Y. B. 1972, Comm. Astrophys. Space Phys., 4, 173

Šuhada, R., Song, J., Böhringer, H., et al. 2010, A\&A, 514, L3

Vanderlinde, K., Crawford, T. M., de Haan, T., et al. 2010, ApJ, 722, 1180

Vikhlinin, A., McNamara, B. R., Forman, W., et al. 1998, ApJ, 502, 558

Vikhlinin, A., Burenin, R. A., Ebeling, H., et al. 2009, ApJ, 692, 1033

Williamson, R., Benson, B. A., High, F. W., et al. 2011, ApJ, 738, 139

Zenteno, A., Song, J., Desai, S., et al. 2011, ApJ, 734, 3 